\title{
Neutrino mass matrix textures: a data-driven approach
}

\author{
E. Bertuzzo, ${ }^{a}$ P.A.N. Machado ${ }^{a, b, c}$ and R. Zukanovich Funchal ${ }^{a, b}$ \\ ${ }^{a}$ Institut de Physique Théorique, \\ CEA-Saclay, 91191 Gif-sur-Yvette, France \\ ${ }^{b}$ Instituto de Física, Universidade de São Paulo, \\ C. P. 66.318, 05315-970 São Paulo, Brazil \\ ${ }^{c}$ TH Division, Physics Department, CERN, \\ CH-1211 Geneva 23, Switzerland \\ E-mail: enrico.bertuzzo@cea.fr, accioly@fma.if.usp.br, \\ zukanov@if.usp.br
}

ABSTRACT: We analyze the neutrino mass matrix entries and their correlations in a probabilistic fashion, constructing probability distribution functions using the latest results from neutrino oscillation fits. Two cases are considered: the standard three neutrino scenario as well as the inclusion of a new sterile neutrino that potentially explains the reactor and gallium anomalies. We discuss the current limits and future perspectives on the mass matrix elements that can be useful for model building.

Keywords: Beyond Standard Model, Neutrino Physics, Standard Model

ARXIV EPRINT: 1302.0653 


\section{Contents}

1 Introduction 1

2 Reconstructing the neutrino mass matrix from experimental data $\quad 2$

3 The standard scenario $\quad 3$

3.1 Correlations among matrix elements 4

3.1.1 Hierarchical case 4

$\begin{array}{lll}3.1 .2 & \text { Quasi-degenerate case } & 7\end{array}$

$\begin{array}{lll}3.1 .3 & \text { Future perspectives } & 8\end{array}$

4 The $3+1$ scenario $\quad 12$

5 Final discussion and conclusion $\quad 15$

$\begin{array}{ll}\text { A Matrix elements squared } & 17\end{array}$

B Complete set of correlation plots for the matrix elements 21

\section{Introduction}

The year 2012 represents a milestone in neutrino physics. Thanks to the measurement of the last mixing angle of the standard neutrino oscillation scenario, $\theta_{13}$, by the reactor experiments Double-CHOOZ [1], Daya-Bay [2] and RENO [3] (after the first positive evidence from accelerators $[4,5]$ ), the mixing in the leptonic sector is starting to shape up. The impact of a rather unexpectedly large mixing angle $\theta_{13}$ is twofold: it promotes the discovery of $\mathrm{CP}$ violation in the neutrino sector to a yet daunting but conceivable task, and at the same time it proves that the description of neutrino oscillation data must involve all three Standard Model neutrino flavors.

Hints of the sensitivity to $\mathrm{CP}$ phases are already showing their first signs when we combine accelerator $\nu_{\mu} \rightarrow \nu_{e}$ with reactor $\bar{\nu}_{e} \rightarrow \bar{\nu}_{e}$ data [8], or when we perform global fits [9-11]. Furthermore, the combined fit of neutrino oscillation data shows for the first time a very precise and almost complete determination of the parameters that enter the standard neutrino oscillation scheme. In fact, in spite of the unknowns (neutrino mass hierarchy, absolute neutrino mass scale, $\mathrm{CP}$ phases and the correct octant for $\theta_{23}$ ) all measured parameters, with the exception of $\sin ^{2} \theta_{23}$, are now so well determined that it is enough to quote them by giving the best fit value with the $1 \sigma$ uncertainty.

However, not all neutrino data can be explained by this standard scenario of three flavor neutrinos. In fact, along the years a number of so-called anomalies have crept into the picture. First, the excess of $\bar{\nu}_{e}$ events in the $\bar{\nu}_{\mu} \rightarrow \bar{\nu}_{e}$ mode observed by the short 
baseline LSND [12, 13] experiment, now also supported by MiniBOONE data [14], gave rise to the long-standing LSND anomaly. Second, the deficit of $\nu_{e}$ compared to expectations observed by the source calibration experiments performed in the gallium radiochemical solar neutrino detectors GALLEX [15-17] and SAGE [18-20]. This is the so-called gallium anomaly. Third, and more recently, a re-evaluation of the reactor $\bar{\nu}_{e}$ flux [21, 22] resulted in an increase of the total flux by $3.5 \%$. While this increase has essentially no impact on the results of long baseline experiments, it induces a deficit of about $5.7 \%$ in the observed event rates for short baseline $(<100 \mathrm{~m})$ reactor neutrino experiments. This problem has been referred to as the reactor antineutrino anomaly [23].

There are attempts in the literature that try to explain some or all of these anomalies by extending the standard picture to include one or more sterile neutrinos [24-26]. These extensions, as a rule, cannot make appearance and disappearance experiments compatible. However, if one disregards the anomaly connected with the appearance experiments LSND/MiniBOONE (for instance, assuming it is not due to oscillations), it is possible to construct a coherent picture of all solar, atmospheric, reactor and accelerator neutrino oscillation data adding one extra sterile neutrino to the standard framework. This constitutes what has been known as the $3+1$ scenario.

Given the current status of the mixing parameters measurements, and in view of the progress expected in the near future, we think it is timely to analyze the possible structures and correlations among the neutrino mass matrix elements that are compatible with data. In this sense, we update refs. [27, 28] using the most recent available data (see also refs. $[29,30]$ for related analyses). However, our analysis will be probabilistic, since we will construct probability distribution functions for each element of the neutrino mass matrix. We also discuss how this might change with better determination of the presently known oscillation parameters, as well as, the Dirac CP-phase $\delta$. We hope this can be helpful to understand better the patterns statistically preferred by data, and serve as a guide for model builders.

We organize our paper as follows. In section 2 we describe how we will proceed for the construction of probability density functions (PDF) for each element of the neutrino mass matrix and what are the assumptions in each case. In section 3 we analyze the possible textures of the mass matrix in the standard scenario, discussing the correlations among matrix elements in the hierarchical and almost degenerate cases. We also discuss the future prospects for better determining these matrix elements with neutrino oscillation and non-oscillation data, and the possible impact on the theory. In section 4 we extend our analysis to include the possibility of a sterile neutrino with mass and mixings allowed by the reactor and gallium anomalies. We discuss what is the mass matrix pattern in this scenario and how different it will be from the standard case. Finally, in section 5, we make our last comments and draw our conclusions.

\section{Reconstructing the neutrino mass matrix from experimental data}

To access the impact of the progress on the determination of the neutrino oscillation parameters in the last year on the knowledge of the low energy effective neutrino mass matrix, 
in a probabilistic way, we will construct a PDF for each element of the mass matrix in the gauge basis,

$$
m_{\alpha \beta}=\sum_{i} m_{i} e^{-i \lambda_{i}} U_{\alpha i}^{*} U_{\beta i}^{*}
$$

with $\alpha, \beta=e, \mu, \tau, U_{\alpha i}$ the elements of the mixing matrix, $m_{i}$ the neutrino masses and $\lambda_{i}$ the Majorana-type CP phases. We will use the most recent available information from the combination of neutrino oscillation data. Without loss of generality we will take $\lambda_{2}=0$ in our parametrization.

With the exception of $\sin ^{2} \theta_{23}$, we will use the following best fit points for the standard mixing parameters $[10]$

$$
\begin{aligned}
\Delta m_{21}^{2} & =(7.50 \pm 0.185) \times 10^{-5} \mathrm{eV}^{2} \\
\sin ^{2} \theta_{12} & =0.30 \pm 0.013 \\
\Delta m_{31}^{2} & =(+2.47 \pm 0.07) \times 10^{-3} \mathrm{eV}^{2} \quad \text { (normal ordering) } \\
\Delta m_{32}^{2} & =(-2.43 \pm 0.06) \times 10^{-3} \mathrm{eV}^{2} \quad \text { (inverted ordering) } \\
\sin ^{2} \theta_{13} & =0.023 \pm 0.0023
\end{aligned}
$$

and assume these parameters to follow a normal distribution with mean at the best fit point and standard deviation equal to the $1 \sigma$ uncertainty. We will take the unknown Dirac-type and Majorana-type CP phases to be flat distributed between 0 and $2 \pi$. Concerning $\sin ^{2} \theta_{23}$, since the distribution cannot be assumed to be normal, we will use the exact distribution extracted from [10].

For the $3+1$ scenario we will fix the squared mass difference between the sterile and the lightest state to two different experimentally allowed values, $\Delta m_{41}^{2}=1.71 \mathrm{eV}^{2}$ and $\Delta m_{41}^{2}=0.95 \mathrm{eV}^{2}$, allowing the corresponding mixing to vary with a flat distribution inside the ranges $\left|U_{e 4}\right|^{2}=8 \times 10^{-3}-4 \times 10^{-2}$ and $\left|U_{e 4}\right|^{2}=8 \times 10^{-3}-2.5 \times 10^{-2}$, respectively.

To construct the PDF of each $m_{\alpha \beta}$ we use a Monte Carlo method. For all the mixing parameters, we generate random numbers according to their assumed distribution, and compute all the elements of $m_{\alpha \beta}$ in each case. In this manner their distribution will be naturally correlated.

Since we do not know the neutrino mass hierarchy, the correct octant for $\theta_{23}$ and the absolute neutrino mass scale, we will have to analyze each case separately.

\section{The standard scenario}

In the standard scenario we use the standard parametrization for the mixing matrix,

$$
U=\left(\begin{array}{ccc}
c_{12} c_{13} & s_{12} c_{13} & s_{13} e^{-i \delta} \\
-s_{12} c_{23}-c_{12} s_{13} s_{23} e^{i \delta} & c_{12} c_{23}-s_{12} s_{13} s_{23} e^{i \delta} & c_{13} s_{23} \\
s_{12} s_{23}-c_{12} s_{13} c_{23} e^{i \delta} & -c_{12} s_{23}-s_{12} s_{13} c_{23} e^{i \delta} & c_{13} c_{23}
\end{array}\right)
$$

with $s_{i j}=\sin \theta_{i j}, c_{i j}=\cos \theta_{i j}$ and $\delta$ the Dirac-type CP phase. The two additional Majorana-type CP phases are denoted by $\lambda_{1}$ and $\lambda_{3}$, and the three neutrino mass eigenstates are ordered either as $m_{1}<m_{2}<m_{3}$ (normal ordering) or as $m_{3}<m_{1}<m_{2}$ (inverted ordering). 


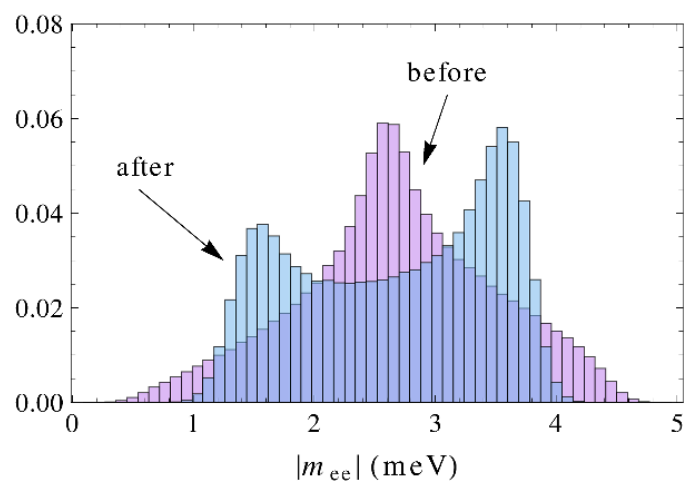

Figure 1. PDF for $\left|m_{e e}\right|$ when $m_{1} \rightarrow 0$. The "before" ("after") distribution corresponds to the situation before (after) the determination of $\sin ^{2} \theta_{13}$ by the reactor experiments.

We will study the following different cases: hierarchical with $m_{1} \rightarrow 0$; hierarchical with $m_{3} \rightarrow 0$; quasi-degenerate with $m_{1} \sim m_{2} \sim m_{3} \sim 0.1 \mathrm{eV}$. There are two possible ordering also in the quasi-degenerate case; however, we have checked that the results are very similar. In all cases, we will show the results for the complete $s_{23}^{2}$ distribution, as well as what is obtained cutting sharply the distribution to force $\theta_{23}$ to lie in the first or second octant.

In figure 1 we illustrate, for the case $m_{1} \rightarrow 0$, the impact of the determination of $\sin ^{2} \theta_{13}$ on the PDF of $\left|m_{e e}\right|$. The distribution labeled "before" (magenta) is obtained assuming $\sin ^{2} \theta_{13}$ to be flat distributed between 0 and 0.04 (CHOOZ limit [31]), while the one labeled "after" (blue) shows the current situation. The two peaks in the "after" distribution are due to the interference between the real $U_{e 2}^{2} m_{2}$ term and the complex $U_{e 3}^{2} m_{3}$ term (see appendix A for detailed expressions), which depends on the cosine of the randomly distributed CP-phases. This term depends on $\theta_{13}$, which gives now a sizable contribution, not being anymore compatible with zero. The distance between the peaks depends on $m_{3}$ : a larger $m_{3}$ would place the peaks further apart.

In figure 2 we illustrate, again for the case $m_{1} \rightarrow 0$, the effect of the determination of $\sin ^{2} \theta_{23}$ (with maximal mixing now disfavored), as opposed to the maximal angle case with MINOS uncertainty $\left(\sin ^{2} \theta_{23}=0.5 \pm 0.1\right.$ [32]). In the two panels we show the case of Normal (left) and Inverted (right) Hierarchy. The asymmetric two peaks structure is due to the fact that $\theta_{12}$ is not maximal. A larger $m_{3}$ would shift the right endpoint of the distribution to higher values of $\left|m_{\mu \mu}\right|$, while a larger $m_{2}$ would separate the two peaks.

\subsection{Correlations among matrix elements}

\subsubsection{Hierarchical case}

In the case of Normal Hierarchy with $m_{1} \rightarrow 0, m_{3} \approx 0.05 \mathrm{eV} \gg m_{2} \approx 0.009 \mathrm{eV}$ and only two CP-phases, $\delta$ and $\lambda_{3}$, are relevant. Due to the $\mu \rightarrow \tau$ symmetry, accomplished by $s_{23} \rightarrow c_{23}$ and $c_{23} \rightarrow-s_{23}$, the PDFs for the solution in the first $\theta_{23}$ octant are basically the same as for the solution in the second octant as long as we replace: $\left|m_{e \tau}\right| \leftrightarrow\left|m_{e \mu}\right|$, $\left|m_{\tau \tau}\right| \leftrightarrow\left|m_{\mu \mu}\right|$. 

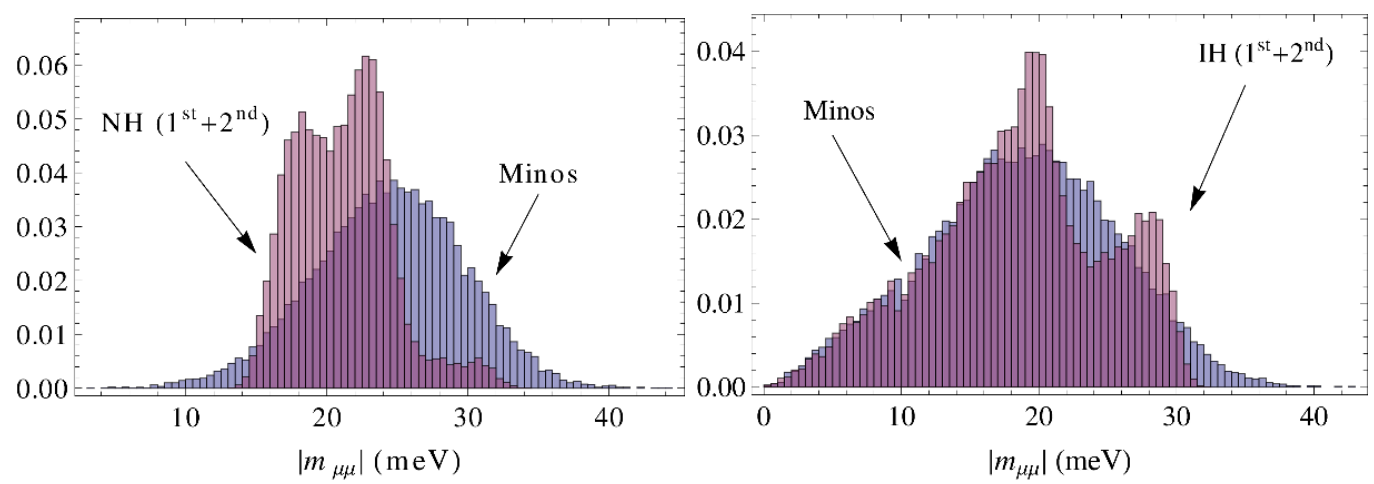

Figure 2. PDF for $\left|m_{\mu \mu}\right|$ for Normal (left panel) and Inverted (right panel) Hierarchy. The distributions are obtained using the $\chi^{2}$ function extracted from [10]. For comparison, in both cases we show the corresponding distribution using MINOS uncertainty [32].

In figure 3 we show the correlations among the absolute values of some of the matrix elements $m_{\alpha \beta}$ for $m_{1} \rightarrow 0$. For $\sin ^{2} \theta_{23}$, the complete $\chi^{2}$ distribution of [10] is used for the colored regions (with blue, green and red referring to the allowed region at $68.27 \%$, 95.45\% and $99.73 \%$ CL, respectively), while the dashed (dotted) lines refer to the $99.73 \%$ allowed region obtained cutting the distribution to force $\theta_{23}$ to lie in the first (second) octant. In appendix B we present a complete set of those plots, obtained constructing a two-dimensional PDF for each pair of elements. In all cases, on the top and to the right of each two-dimensional distribution the projected PDFs are shown. The range of the values allowed at $95.45 \%$ CL are given in table 1.

From figures 3 and 13 we observe that $\left|m_{e e}\right|$ is not very correlated to any other element. However, due to $\theta_{23}$ the pairs $\left|m_{e \mu}\right| \times\left|m_{e \tau}\right|,\left|m_{e \tau}\right| \times\left|m_{\mu \mu}\right|,\left|m_{\mu \mu}\right| \times\left|m_{\mu \tau}\right|,\left|m_{\mu \tau}\right| \times\left|m_{\tau \tau}\right|$, $\left|m_{\mu \mu}\right| \times\left|m_{\tau \tau}\right|$ and $\left|m_{e \mu}\right| \times\left|m_{\tau \tau}\right|$ are very correlated. So, for instance, if a model predicts $\left|m_{e \mu}\right| \approx 6 \mathrm{meV},\left|m_{e \tau}\right|$ must be in the range $5-8 \mathrm{meV}$, while without taking into account this correlation the allowed range would be $2.7-9.3 \mathrm{meV}$ at $95.45 \%$ CL.

From the expressions for the matrix elements given in appendix A it is easy to show that in this case:

$$
\begin{aligned}
\left|m_{e \mu}\right|^{2}+\left|m_{e \tau}\right|^{2} & \sim m_{3}^{2} y^{2}+m_{2}^{2} x^{2}, \\
\left|m_{\mu \mu}\right| & \sim m_{3} z^{2}, \\
\left|m_{\tau \tau}\right|^{2} & \sim m_{3}^{2}\left(1-2 z^{2}\right)+\left|m_{\mu \mu}\right|^{2},
\end{aligned}
$$

and

$$
\left|m_{\mu \tau}\right|^{2}+\left|m_{\tau \tau}\right|^{2} \sim m_{3}^{2}\left(1-z^{2}\right),
$$

where $x=\sin \theta_{12}, y=\sin \theta_{13}$ and $z=\sin \theta_{23}$.

Due to the prevalence of the $m_{3}$ mass, the determination of $\sin ^{2} \theta_{13}$ with the present uncertainty of $10 \%$ by the reactor experiments not only affected the range of $\left|m_{e \alpha}\right|, \alpha=$ $e, \mu, \tau$, but also their PDFs.

In the case of Inverted Hierarchy with $m_{3} \rightarrow 0, m_{1} \approx m_{2} \approx 0.05 \mathrm{eV}$ and only two CP-phases, $\delta$ and $\lambda_{1}$, are important. In figure 4 we show two-dimensional PDFs for some 

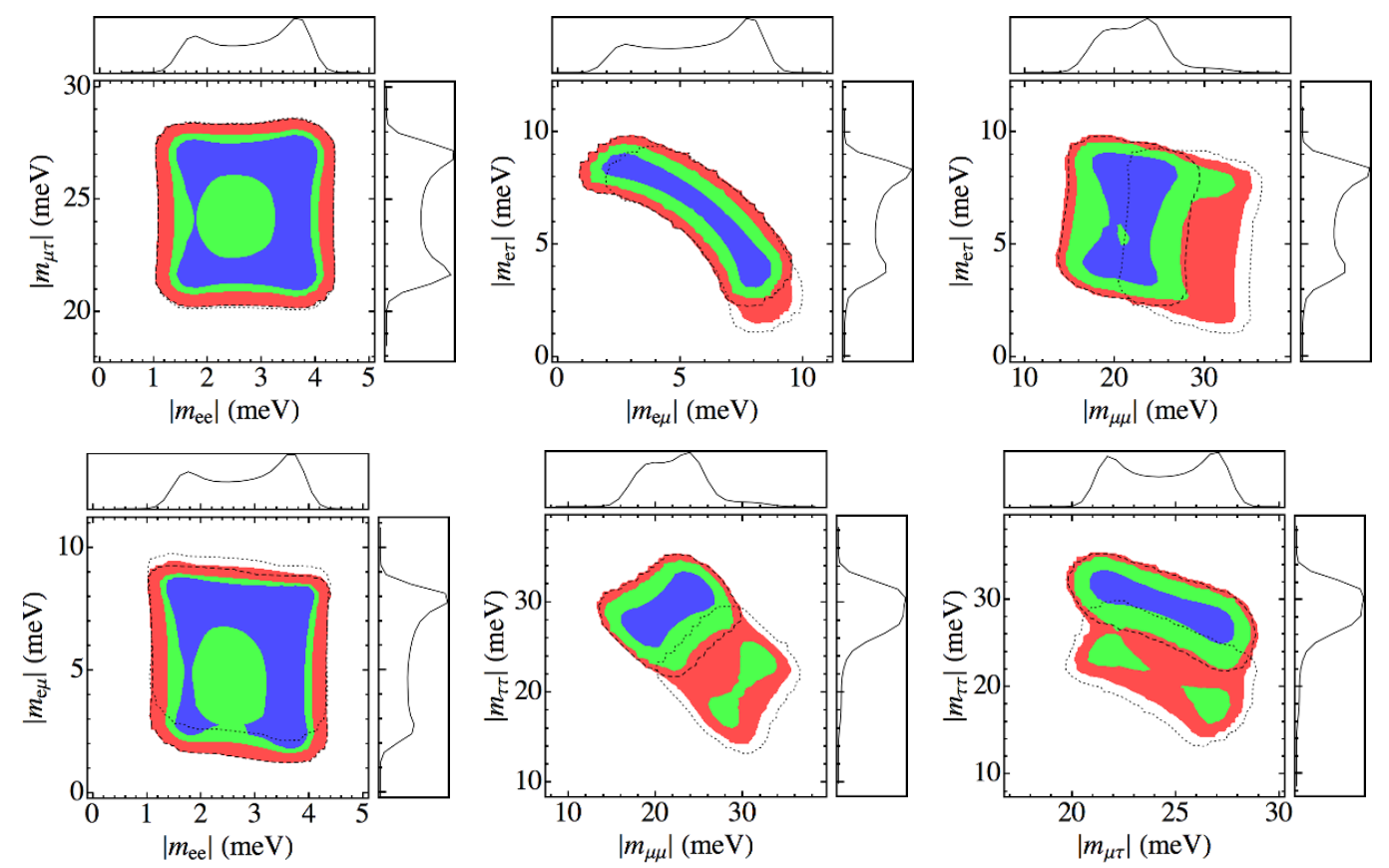

Figure 3. PDFs for the distribution of the absolute value of several pairs of matrix elements for Normal Ordering and $m_{1} \rightarrow 0$. Top panels: $\left|m_{e e}\right| \times\left|m_{\mu \tau}\right|$ (left), $\left|m_{e \mu}\right| \times\left|m_{e \tau}\right|$ (center) and $\left|m_{\mu \mu}\right| \times\left|m_{e \tau}\right|$ (right). Bottom panels: $\left|m_{e e}\right| \times\left|m_{e \mu}\right|$ (left), $\left|m_{\mu \mu}\right| \times\left|m_{\tau \tau}\right|$ (center) and $\left|m_{\mu \tau}\right| \times\left|m_{\tau \tau}\right|$ (right). At the top and right of each two dimensional PDF we show the PDF of the absolute value of the corresponding matrix element. The colored regions refer to the allowed regions at $68.27 \%$ (blue), $95.45 \%$ (green) and $99.73 \%$ CL (red), as obtained using the complete $\chi^{2}$ distribution for $\sin ^{2} \theta_{23}$. The dashed (dotted) lines refer instead to the $95.45 \%$ allowed region obtained for $\theta_{23}$ in the first (second) octant (see text).

\begin{tabular}{|l|c|c|}
\hline \multicolumn{3}{|c|}{ in $\mathrm{meV}$} \\
\hline Element & $m_{1} \rightarrow 0$ & $m_{3} \rightarrow 0$ \\
\hline$\left|m_{e e}\right|$ & $1.3-4.1$ & $19-52$ \\
$\left|m_{e \mu}\right|$ & $1.9-8.4$ & $4.4-37$ \\
$\left|m_{e \tau}\right|$ & $3.0-8.9$ & $4.7-37$ \\
$\left|m_{\mu \mu}\right|$ & $15-29$ & $6.2-31$ \\
$\left|m_{\mu \tau}\right|$ & $21-28$ & $9.9-26$ \\
$\left|m_{\tau \tau}\right|$ & $22-34$ & $7.1-32$ \\
\hline
\end{tabular}

Table 1. Range of allowed values of $\left|m_{\alpha \beta}\right|$ at $95.45 \%$ CL for the very hierarchical cases.

pairs of elements of the matrix $m_{\alpha \beta}$ in this case. The complete set of plots can be found in appendix B, while in table 1 we present the $95.45 \%$ CL allowed ranges.

Generally, the dominant terms comprise $m_{1}$ or $m_{2}$, which have similar sizes, and their contributions involve $\theta_{12}$ and $\theta_{23}$, which are not maximal, without being suppressed by $\theta_{13}$. There are at least three consequences of such a fact. First, the determination of $\sin ^{2} \theta_{13}$ by the reactor experiments basically did not affect the range of the matrix elements but changed the shape of some of their PDFs. Second, the determination of $\sin ^{2} \theta_{23}$ with an 

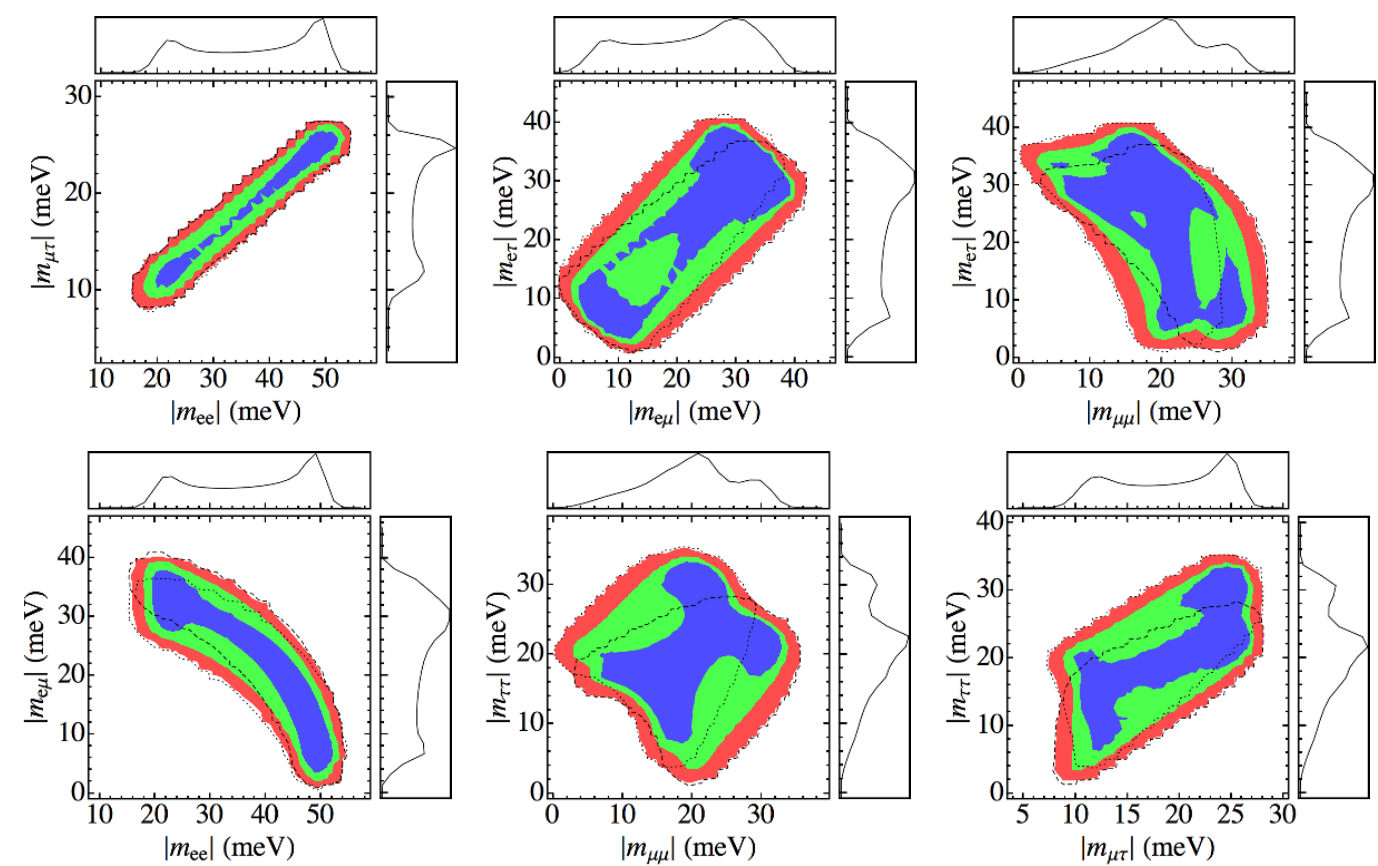

Figure 4. Same as figure 3 but for Inverted Ordering with $m_{3} \rightarrow 0$.

uncertainty of $9 \%$ changes the range $\left|m_{e \mu}\right|,\left|m_{e \tau}\right|,\left|m_{\mu \mu}\right|$ and $\left|m_{\tau \tau}\right|$, while the shape of the PDFs remain basically the same except in the case $\left|m_{\mu \mu}\right|$ and $\left|m_{\tau \tau}\right|$. Last, the mass matrix entries are very correlated.

We observe the strong correlations between all pairs of elements in figures 4 and 14. As a consequence, in this case it is even more important to take into account these correlations in model building. For instance, we can easily see, from the expressions in appendix A, that in this case

$$
\begin{aligned}
\left|m_{\mu \tau}\right| & \sim \sqrt{z^{2}\left(1-z^{2}\right)}\left|m_{e e}\right| \\
\left|m_{e e}\right|^{2} & \sim m_{2}^{2}-\left(1-z^{2}\right)^{-1}\left|m_{e \mu}\right|^{2}
\end{aligned}
$$

and

$$
\left|m_{e \mu}\right| \sim \sqrt{\frac{\left(1-z^{2}\right)}{z^{2}}}\left|m_{e \tau}\right| .
$$

This behavior is confirmed by figure 4 .

\subsubsection{Quasi-degenerate case}

In the quasi-degenerate case, $m_{1} \sim m_{2} \sim m_{3}$ and the effect of the ordering is very small. In this case, all masses and $\mathrm{CP}$ phases play a role.

As an example, we take $m_{1}=0.1 \mathrm{eV}$. In figure 5 we show the correlations among the absolute values of some of the matrix elements $m_{\alpha \beta}$ for the normal mass ordering. In appendix B one can find the complete set of plots for this case (figure 15). We use the same color coding as in previous figures. The range of the values allowed at $95.45 \% \mathrm{CL}$ are given in table 2 . 


\begin{tabular}{|l|c|}
\hline \multicolumn{2}{|c|}{ in $\mathrm{meV}$} \\
\hline Element & $m_{1}=0.1 \mathrm{eV}$ \\
\hline$\left|m_{e e}\right|$ & $39-108$ \\
$\left|m_{e \mu}\right|$ & $11-88$ \\
$\left|m_{e \tau}\right|$ & $11-78$ \\
$\left|m_{\mu \mu}\right|$ & $17-113$ \\
$\left|m_{\mu \tau}\right|$ & $21-111$ \\
$\left|m_{\tau \tau}\right|$ & $31-113$ \\
\hline
\end{tabular}

Table 2. Range of allowed values of $\left|m_{\alpha \beta}\right|$ at $95.45 \%$ CL for the quasi-degenerate case with $m_{1}=0.1 \mathrm{eV}$.
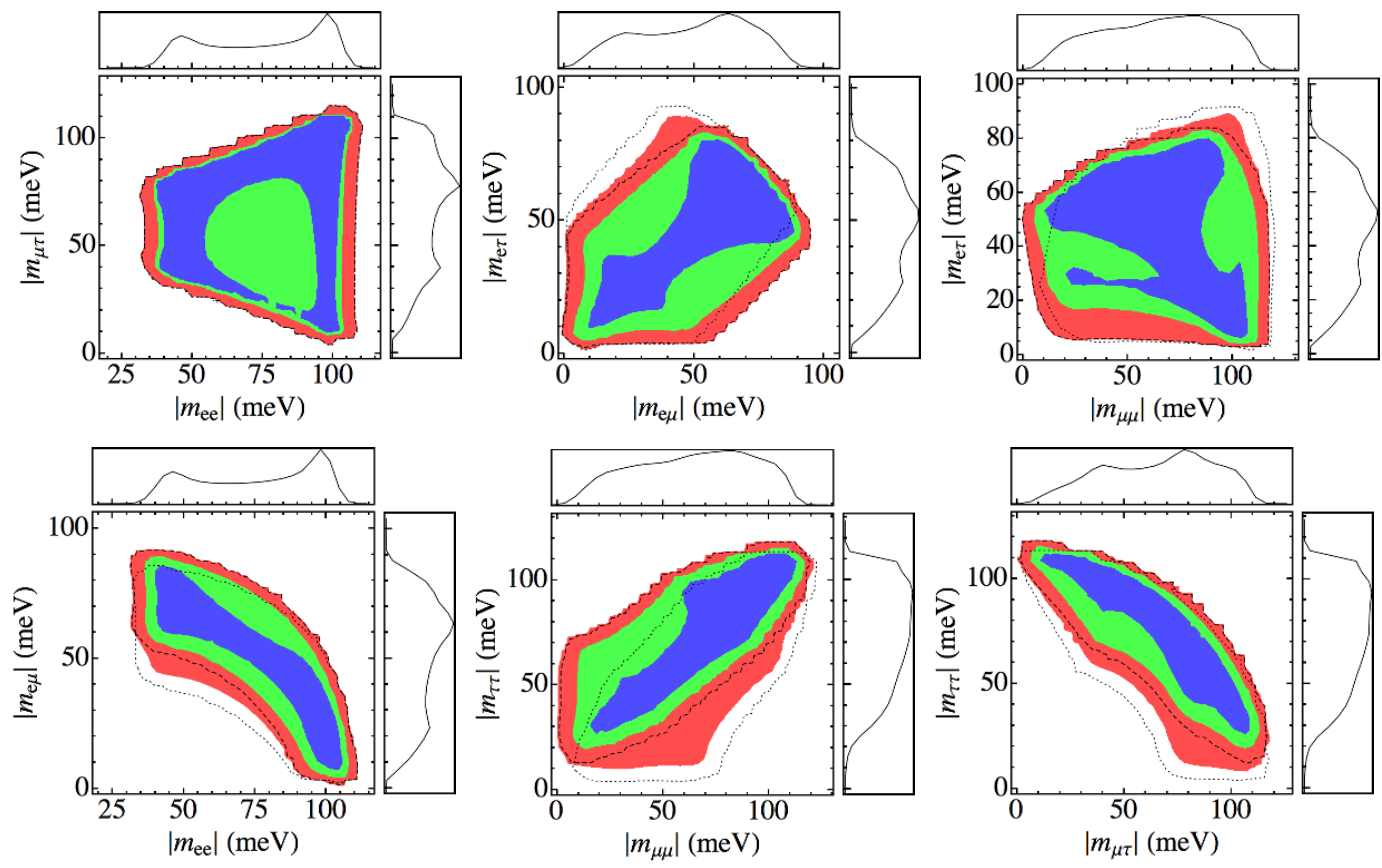

Figure 5. Same as figure 3 but for $m_{1}=0.1 \mathrm{eV}$.

The correlations here are either similar to the very hierarchical case in normal ordering or in the inverted ordering. For example, the PDFs for $\left|m_{e e}\right| \times\left|m_{e \mu}\right|,\left|m_{\mu \mu}\right| \times\left|m_{\tau \tau}\right|,\left|m_{\mu \mu}\right| \times$ $\left|m_{e \tau}\right|$ and $\left|m_{e \mu}\right| \times\left|m_{e \tau}\right|$, are correlated like in the inverted ordering, while $\left|m_{\mu \tau}\right| \times\left|m_{\tau \tau}\right|$ and $\left|m_{e e}\right| \times\left|m_{\mu \tau}\right|$ are more like the normal ordering.

\subsubsection{Future perspectives}

To evaluate the effect of a future determination of the mixing parameters on our knowledge of the mass matrix we have studied the effect of reducing the uncertainty of each parameter at a time while keeping the other parameters at their current uncertainties. We assume that the following uncertainties will be achieved by the present or next generation experiments, at $68 \% \mathrm{CL}$. Accelerators experiments like for example T2K will be able to measure $\delta\left(\sin ^{2} 2 \theta_{23}\right) \approx 0.01$ [6]. Possible medium baseline reactor experiments 
could, in principle, determine $\delta\left(\Delta m_{31}^{2}\right) \approx 7 \times 10^{-6} \mathrm{eV}^{2}, \delta\left(\Delta m_{21}^{2}\right) \approx 3 \times 10^{-7} \mathrm{eV}^{2}$, and $\delta\left(\sin ^{2} \theta_{12}\right) \approx 0.004$ [7]. Within the current systematic uncertainties, the Daya Bay experiment can probe $\delta\left(\sin ^{2} \theta_{13}\right) \approx 0.0013[2]$.

In figure 6 we illustrate the effect of a better determination of $\sin ^{2} \theta_{13}$ (top left panel), $\Delta m_{31}^{2}$ (top right panel), $\sin ^{2} \theta_{21}$ (bottom left panel) and $\sin ^{2} \theta_{23}$ (bottom right panel). We observe that the effect of a better determination of any of these parameters is very small. We have verified that this is true for both mass hierarchies and $\theta_{23}$ octants. The biggest effect comes from a better determination of $\sin ^{2} \theta_{23}$, as one could have guessed, but still this only reduces significantly the $3 \sigma$ region. We do not show the effect of a better determination of $\Delta m_{21}^{2}$ because it is even smaller than for the other parameters.

On the other hand, a measurement of $\delta$ with an uncertainty of $10^{\circ}$, that can be envisaged according to ref. [38] for long baseline neutrino oscillation experiments, could be significant. To illustrate this we show in figures 7-8 the effect of a determination of $\delta$ with an uncertainty of $10^{\circ}$ for some of the correlations between pairs of mass matrix elements. On the top (bottom) panels of figure 7 we can see this for $\left|m_{e e}\right| \times\left|m_{e \mu}\right|\left(\left|m_{\mu \mu}\right| \times\left|m_{e \tau}\right|\right)$ in the normal ordering for $\delta=0^{\circ}$ (left), $180^{\circ}$ (center) and $270^{\circ}$ (right), while on the top (bottom) panels of figure 8 we show $\left|m_{\mu \tau}\right| \times\left|m_{\tau \tau}\right|\left(\left|m_{\mu \mu}\right| \times\left|m_{e \tau}\right|\right)$ in the inverted ordering for $\delta=0^{\circ}$ (left), $90^{\circ}$ (center) and $180^{\circ}$ (right), These cases for normal (inverted) ordering and $\delta=90^{\circ}\left(\delta=270^{\circ}\right)$ are not shown because they are very similar to $\delta=270^{\circ}\left(\delta=90^{\circ}\right)$.

For the normal ordering, with $m_{1} \rightarrow 0$, the determination of $\delta$ will play an important role in the correlation of $\left|m_{e e}\right|$ with all other mass matrix elements, but will be more significant for $\left|m_{e \mu}\right|$ or $\left|m_{e \tau}\right|$. This is due to the fact that for these mass matrix elements the leading phase term is the one that accompanies $\cos \left[2\left(\delta+\lambda_{3}\right)\right]$, just as for $\left|m_{e e}\right|$. This will also affect the correlations involving $\left|m_{\mu \mu}\right|$ and $\left|m_{\tau \tau}\right|$, since for them the leading phase terms are, in order of importance, the ones that go with $\cos \left(2 \lambda_{3}\right)$ and $\cos \left(\delta+2 \lambda_{3}\right)$. However, the correlations with $\left|m_{\mu \tau}\right|$ will only slightly change because the leading phase term for this element does not depend on $\delta$.

For the inverted ordering, with $m_{3} \rightarrow 0$, the determination of $\delta$ will play a bigger role in the PDFs of $\left|m_{\tau \tau}\right|$ and $\left|m_{\mu \mu}\right|$. This is because, as we can see from their expressions in appendix A, the leading coefficients of $\cos \left(2 \lambda_{1}\right), \cos \left(\delta \pm 2 \lambda_{1}\right)$ and $\cos \delta$ are all of the same order. The PDFs of $\left|m_{e \mu}\right|$ and $\left|m_{e \tau}\right|$ will also be affected because the terms that depend on $\delta$ are not negligible in comparison to the leading term that depends on $\cos \left(2 \lambda_{1}\right)$, however their relative importance will depend on the $\theta_{23}$ octant. The PDFs for $\left|m_{e e}\right|$ and $\left|m_{\mu \tau}\right|$ are basically independent of $\delta$, the first because $m_{3} \rightarrow 0$, the second because these terms are suppressed by $\sin ^{2} \theta_{13}$ or factors of this order.

We also have checked that the effect of the determination of $\delta$ for the quasi-degenerate case with $m_{1}=0.1 \mathrm{eV}$ is smaller than for the hierarchical cases because there are more phases involved.

In the future we also expect to have information from three different sources: neutrinoless double beta decay experiments, beta decay experiments and cosmology. In figure 9 we show the current allowed region for $\left|m_{e e}\right|$ as a function of the effective electron neutrino 
mass, $m_{\beta}$ and of the sum of the neutrino masses, ${ }^{1} \sum m_{i}$ at $99 \% \mathrm{CL}$. The region allowed by the normal (inverted) mass ordering is in blue (red) and the recent limit on $\left|m_{e e}\right|$ given by KamLAND-Zen [39], $\left|m_{e e}\right|<(120-250) \mathrm{meV}$, is shown in gray. Cosmology excludes the magenta region $\sum m_{i}>(0.2-0.6) \mathrm{eV}$ [41]. Notice that the allowed regions were built from the pdfs constructed from data (except for the CP phases, which we assumed to be flat distributed). This is why the $m_{e e} \rightarrow 0$ region is absent, as it is very unlikely to have the necessary degree of cancellations.

The forecast sensitivity on $\left|m_{e e}\right|$ of the most ambitious neutrinoless double beta decay experiments, after 5 years of exposure, is $29-73 \mathrm{meV}$ (GERDA phase-3) and 18-39 meV (CUORE) [40].

The KArlsruhe TRitium Neutrino mass experiment (KATRIN) will have a sensitivity on the electron neutrino effective mass, $m_{\beta}=\sqrt{\sum_{i}\left|U_{e i}\right|^{2} m_{i}^{2}}$, of $0.2 \mathrm{eV}$ at $95 \% \mathrm{CL}$ [42].

Cosmological limits today still allow for quasi-degenerate neutrino masses; however, this possibility will soon be confirmed or ruled out. According to ref. [41], many cosmological probes, with different systematics, will reach a sensitivity on $\sum m_{i}$ of $0.1 \mathrm{eV}$ or lower. Lyman $\alpha$ forest can reach $0.1 \mathrm{eV}$, lensing of Cosmic Microwave Background 0.2$0.05 \mathrm{eV}$, lensing of galaxies $0.07 \mathrm{eV}$, observations of the redshifted $21 \mathrm{~cm}$ neutral hydrogen line $0.1-0.006 \mathrm{eV}$, galaxy clusters and galaxy distribution surveys $0.1 \mathrm{eV}$.

If cosmology will point to a quasi-degenerate neutrino spectrum:

1. the determination of $\delta$ by future experiments will not modify much the current correlation among mass matrix elements;

2. $\left|m_{e e}\right|$ should be in the reach of most proposed neutrinoless double beta decay experiments, if neutrinos are of Majorana nature, ergo the non-observability of the $0 \nu \beta \beta$ would point to Dirac neutrinos;

3. $m_{\beta}$ may be in the reach of KATRIN;

4. the ordering of neutrino masses can be settled by future neutrino oscillation experiments but will not have great impact on the neutrino mass matrix;

5. if $\left|m_{e e}\right|$ is measured we will also be able to constrain much more $\left|m_{e \mu}\right|$ and $\left|m_{e \tau}\right|$. However, it will be very difficult to say something about the Majorana-CP phases.

On the other hand, cosmology can also place a limit on $\sum m_{i}$, such that we will know if we have normal ordering with $m_{1} \rightarrow 0$. In this case:

1. $\left|m_{e e}\right|$ will be out of the reach of the proposed $0 \nu \beta \beta$ experiments;

2. $m_{\beta}$ will be out of the reach of KATRIN;

3. the experimental determination of $\delta$ will increase the correlation among mass matrix elements and help to determine the structure of the neutrino mass matrix;

\footnotetext{
${ }^{1}$ There is no one-to-one correspondence between $m_{e e}$ and $m_{\beta}$ or $\sum_{i} m_{i}$. Hence, in order to plot figure 9, for each value of $m_{0}$ we extracted the allowed interval of $m_{e e}$ and plotted it against $m_{\beta}$ and $\sum_{i} m_{i}$ calculated at the best fit values of the oscillation parameters (as the Majorana phases do not play a role in these last two quantities).
} 
4. the ordering of neutrino masses should be confirmed by future neutrino oscillation experiments.

It may be the case that cosmology will rule out a quasi-degenerate spectrum, but not the inverted ordering. If this happens:

1. $\left|m_{e e}\right|$ may be in the reach of the proposed $0 \nu \beta \beta$ experiments;

2. $m_{\beta}$ will be out of the reach of KATRIN;

3. the ordering of neutrino masses should be determined by future neutrino oscillation experiments;

4. the experimental determination of $\delta$ will increase the correlation among mass matrix elements and help to determine the structure of the neutrino mass matrix, specially if the mass ordering is known;

5. we may be able to say something about one of the Majorana CP phases, if $\left|m_{e e}\right|$ is measured.

These future advances may thus provide new clues for the understanding of the flavor problem in the lepton sector. Some models of neutrino mixing based on discrete flavor groups have predictions that can be tested in the future. For instance, the Lin model [33], where the $A_{4}$ symmetry is broken by additional $Z_{n}$ parities, predicts

$$
\sin ^{2} \theta_{23}=\frac{1}{2}+\frac{1}{\sqrt{2}} \sin \theta_{13} \cos \delta
$$

Another example is the SUSY model based on the flavor symmetry group $S_{4} \times Z_{4} \times \mathrm{U}(1)$ discussed in [34] where the relation

$$
\sin ^{2} \theta_{12}=\frac{1}{2}+\sin \theta_{13} \cos \delta+\mathcal{O}\left(\sin ^{2} \theta_{13}\right)
$$

appears. Both relations can, in principle, be experimentally tested and, if true, they will impose new correlations among the neutrino mass matrix elements.

Other models, such as the one presented in ref. [35], are even more predictive. This model, which is based on a type-I seesaw framework with an underlying $A_{4}$ flavor symmetry, can, given a set of vacuum expectation value alignments for the flavon fields which break the $A_{4}$ symmetry, predict neutrino masses, the mass hierarchy, $\theta_{23}$ and $\delta$.

There are also model-independent approaches to the flavor problem in the neutrino sector. In ref. [36, 37], relations among the mixing parameters were obtained, in the context of discrete flavor symmetries, under general assumptions that the flavor symmetry group is of the von Dyck type. Again these relations can, in principle, be experimentally tested, and if ratified by experiment induce more correlations among the mixing matrix entries. 

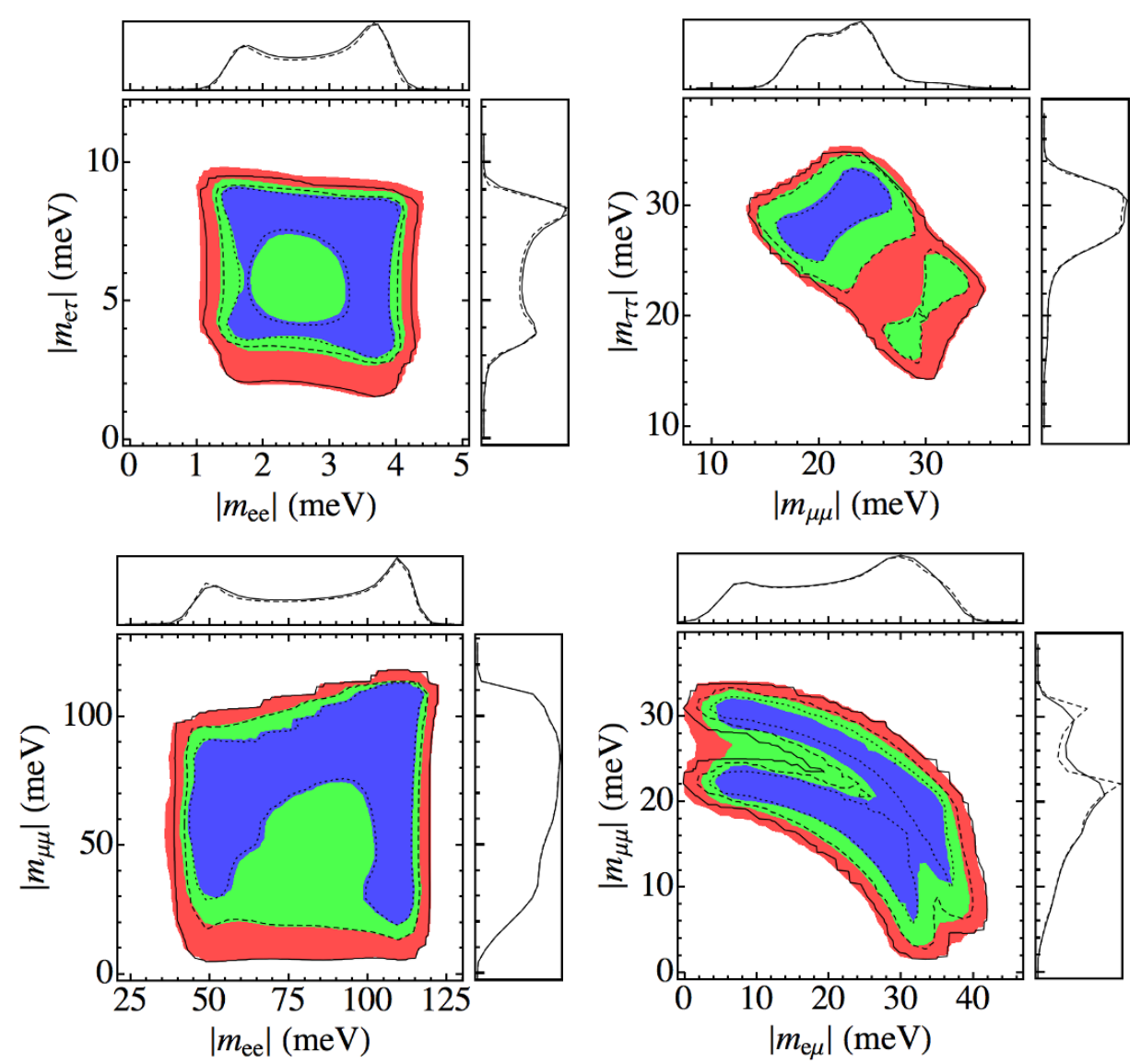

Figure 6. PDFs for the distribution of: $\left|m_{e e}\right| \times\left|m_{e \tau}\right|$ for the the normal hierarchy with $\sin ^{2} \theta_{13}$ uncertainty reduced (top left panel); $\left|m_{\mu \mu}\right| \times\left|m_{\tau \tau}\right|$ for the the normal hierarchy with $\Delta m_{31}^{2}$ uncertainty reduced (top right panel); $\left|m_{e e}\right| \times\left|m_{\mu \mu}\right|$ for the the quasi-degenerate case with $\sin ^{2} \theta_{12}$ uncertainty reduced (bottom left panel) and $\left|m_{e \mu}\right| \times\left|m_{\mu \mu}\right|$ for the the inverted hierarchy with $\sin ^{2} \theta_{23}$ uncertainty reduced (bottom right panel). The colored areas are for the present uncertainties of the oscillation parameters, whereas the back lines are for the assumed future reduced uncertainty of one of the parameters (see text for details).

\section{The $3+1$ scenario}

Whether or not one deems this to be a plausible scenario, we still believe it is important to examine what are its consequences to the possible textures of the neutrino mass matrix.

In this case the mixing matrix can be parametrized as

$$
U=\left(\begin{array}{cccc}
c_{12} c_{13} c_{14} & s_{12} c_{13} c_{14} & s_{13} c_{14} e^{-i \delta} & s_{14} \\
-s_{12} c_{23}-c_{12} s_{13} s_{23} e^{i \delta} & c_{12} c_{23}-s_{12} s_{13} s_{23} e^{i \delta} & c_{13} s_{23} & 0 \\
s_{12} s_{23}-c_{12} s_{13} c_{23} e^{i \delta} & -c_{12} s_{23}-s_{12} s_{13} c_{23} e^{i \delta} & c_{13} c_{23} & 0 \\
-c_{12} c_{13} s_{14} & -s_{12} c_{13} s_{14} & -s_{13} s_{14} e^{-i \delta} & c_{14}
\end{array}\right),
$$

where we use the same notation as in eq. (3.1). This expression can be readily derived from [44] once we identify $\theta_{s} \equiv \theta_{14}$ and we assume $\vec{n}=(1,0,0)$, i.e. the sterile state mixes only with the electron neutrino. With this assumptions, there are no extra Dirac CP 

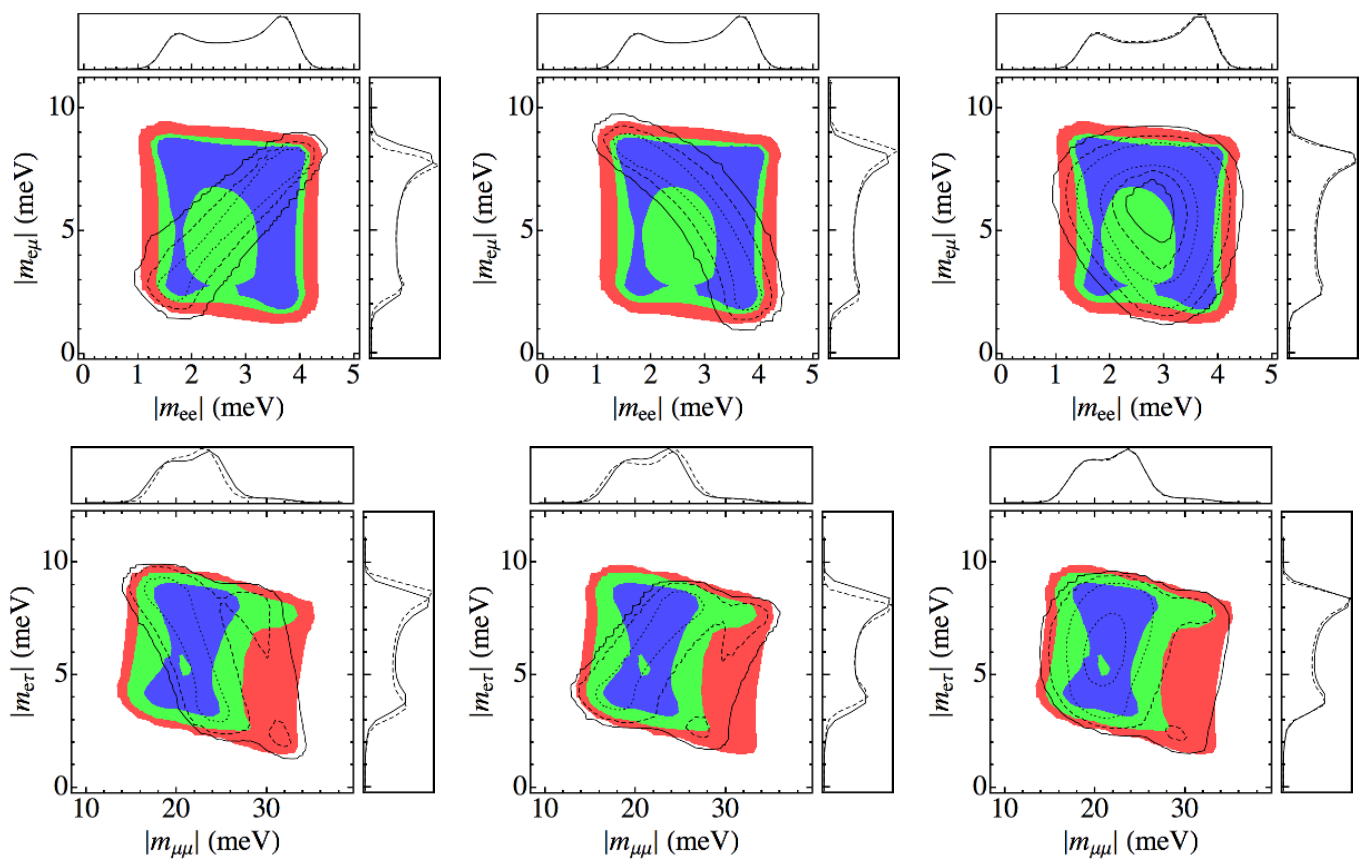

Figure 7. PDFs for the distribution of: $\left|m_{e e}\right| \times\left|m_{e \mu}\right|$ (top panels) and $\left|m_{\mu \mu}\right| \times\left|m_{e \tau}\right|$ (bottom panels) for the normal ordering. From left to right $\delta=0^{\circ}, 180^{\circ}$ and $270^{\circ}$, assumed to be determined within $10^{\circ}$.
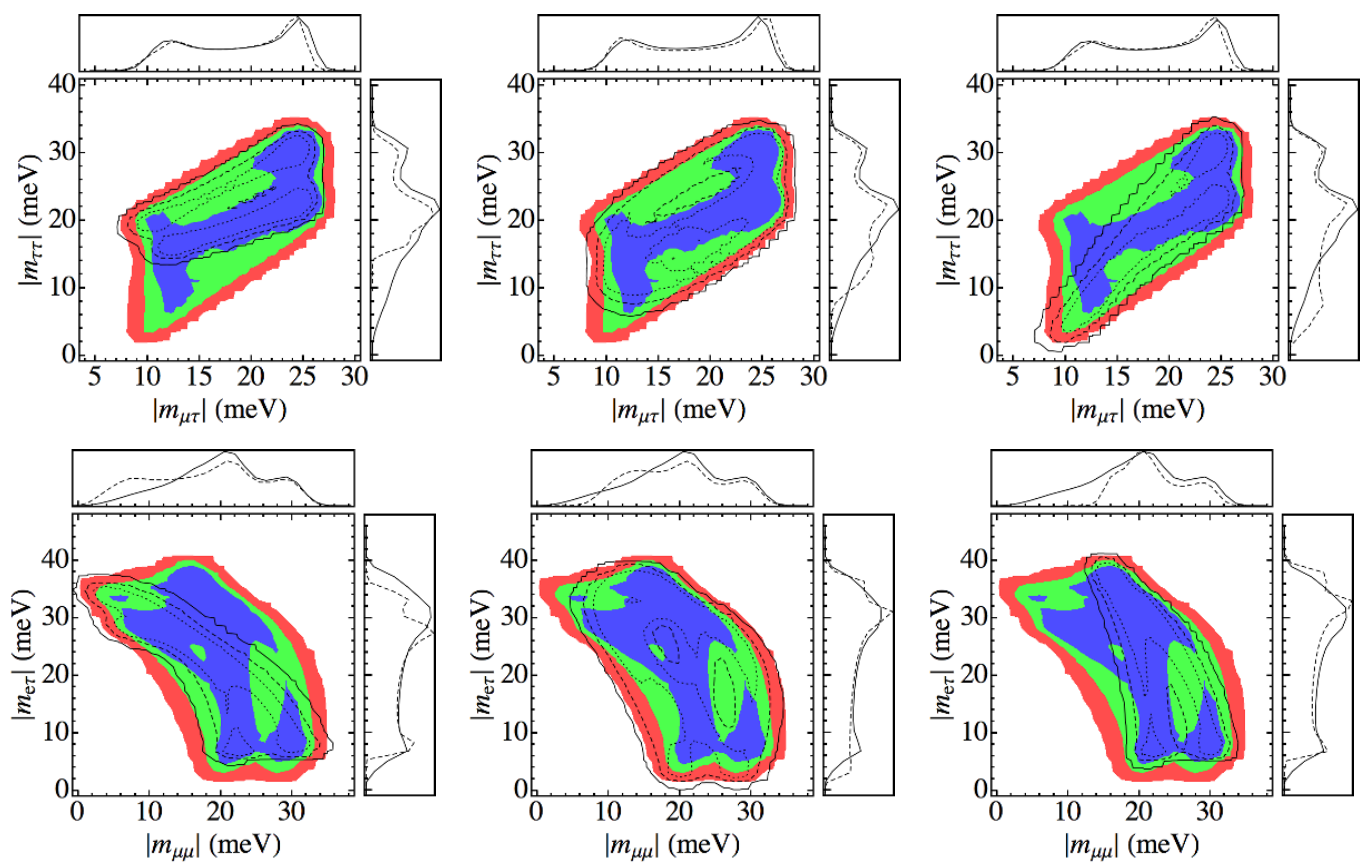

Figure 8. PDFs for the distribution of: $\left|m_{\mu \tau}\right| \times\left|m_{\tau \tau}\right|$ (top panels) and $\left|m_{\mu \mu}\right| \times\left|m_{e \tau}\right|$ (bottom panels) for the inverted ordering. From left to right $\delta=0^{\circ}, 90^{\circ}$ and $180^{\circ}$, assumed to be determined within $10^{\circ}$.

phases. Nevertheless, there is one extra Majorana phase, $\lambda_{4}$. The expression of all the squared matrix elements, in the limit $c_{13} \sim c_{14} \rightarrow 1$, can be found in appendix A. 

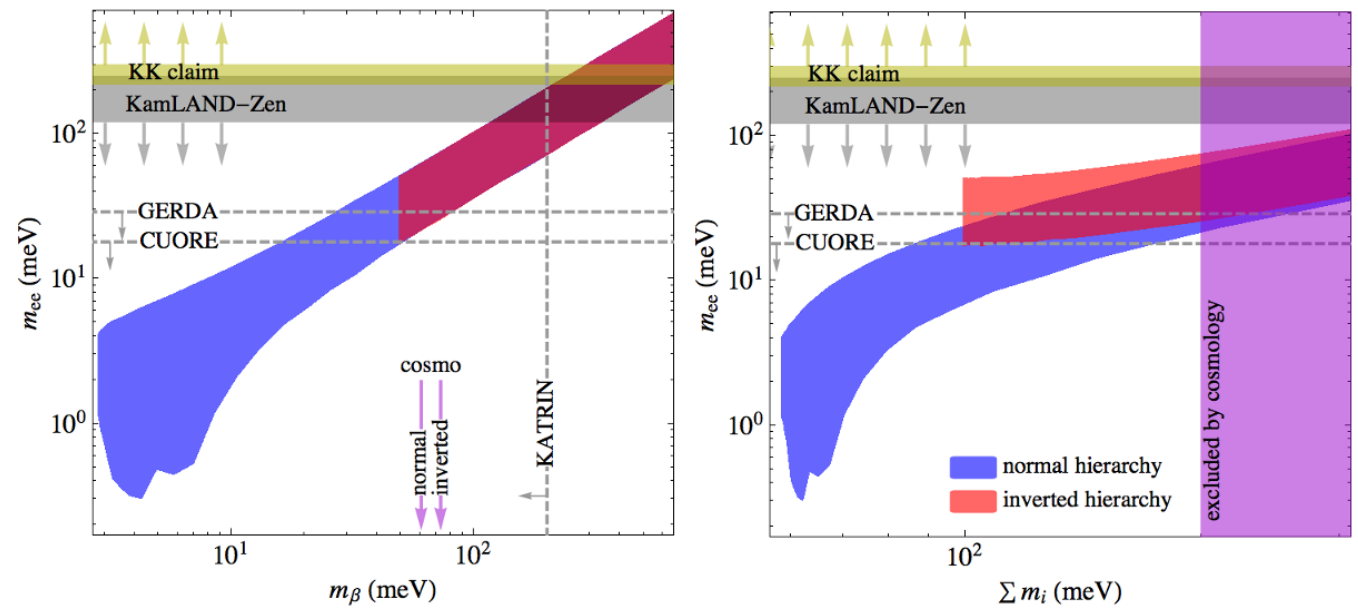

Figure 9. We show the current allowed regions for $\left|m_{e e}\right|$ at $99 \%$ CL as a function of the effective electron neutrino mass, $m_{\beta}$, on the left panel and as a function of the sum of the neutrino masses, $\sum m_{i}$, on the right panel. The region allowed by the normal (inverted) mass ordering is in blue (red), the recent limit on $\left|m_{e e}\right|$ given by KamLAND-Zen [39] in gray and the region excluded by cosmology [41] in magenta. We also show the reach expected for the beta decay experiment Katrin [42], as well as the ultimate reach aimed by the neutrinoless double beta decay experiments GERDA and CUORE according to ref. [40].

For simplicity, we examine here two cases: (a) $\Delta m_{41}^{2}=1.71 \mathrm{eV}^{2}$ and $\sin ^{2} \theta_{14}=(0.8-$ $4.2) \times 10^{-2}$, (b) $\Delta m_{41}^{2}=0.95 \mathrm{eV}^{2}$ and $\sin ^{2} \theta_{14}=(0.8-2.5) \times 10^{-2}$, which are two possible solutions to the reactor and gallium anomalies [23]. These solutions seem at first glance to be at odds with cosmology, but we will ignore this fact at this point. Since $\sin ^{2} \theta_{14}$ is small, we do not expect big changes in the PDFs of $\left|m_{\alpha \beta}\right|, \alpha, \beta=e, \mu, \tau$, except for the case $\left|m_{e e}\right|$. We have explicitly checked that this is the case.

In figure 10 we show $\left|m_{e e}\right|$ and the new entries $\left|m_{\alpha s}\right|, \quad \alpha=e, \mu, \tau$ for the normal hierarchy and $\Delta m_{41}^{2}=1.71 \mathrm{eV}^{2}$. For the case $\Delta m_{41}^{2}=0.95 \mathrm{eV}^{2}$, the plots would be similar in shape, however with different scale. For $\left|m_{e e}\right|$ the largest value goes down from $0.06 \mathrm{eV}$ to $0.045 \mathrm{eV}$. For $\left|m_{e s}\right|$ the range changes from $\sim(0.12-0.26) \mathrm{eV}$ to $\sim(0.09-0.20) \mathrm{eV}$. For $\left|m_{\mu s}\right|,\left|m_{\tau s}\right|$ there is basically no difference and for $\left|m_{s s}\right|$ there is again a shift in the range from $\sim(1.25-1.30) \mathrm{eV}$ to $\sim(0.935-0.97) \mathrm{eV}$.

In figure 11 we show the correlations among the PDF's of $\left|m_{e e}\right|$ and $\left|m_{e s}\right|,\left|m_{\mu s}\right|,\left|m_{\tau s}\right|$, $\left|m_{s s}\right|$, for the normal hierarchy and $\Delta m_{41}^{2}=1.71 \mathrm{eV}^{2}$. Since the difference between the case $\Delta m_{41}^{2}=1.71 \mathrm{eV}^{2}$ and $\Delta m_{41}^{2}=0.95 \mathrm{eV}^{2}$ is basically the scale, as commented above, we do not show the correlations in this case either.

We observe that $\left|m_{e e}\right|$ is very correlated with $\left|m_{e s}\right|$. This is easy to understand from the formulae in appendix $\mathrm{A}$, as $\left|m_{e e}\right|$ goes as $s_{14}^{2}$ while $\left|m_{e s}\right|$ goes like $s_{14}$ producing a squared root behavior. The thickness is driven by the $\mathrm{CP}$ phases. The linear behavior between $\left|m_{e e}\right|$ and $\left|m_{s s}\right|$ can be explained by noting that $\left|m_{s s}\right| \approx m_{4}-\left|m_{e e}\right|$. Again due to the approximate $\mu-\tau$ symmetry we get similar ranges and behaviors for $\left|m_{e e}\right| \times\left|m_{\mu s}\right|$ and $\left|m_{e e}\right| \times\left|m_{\tau s}\right|$.

Also in this case it is interesting to consider future perspectives, in particular considering the interplay between oscillation experiments and cosmology. From the cosmology 

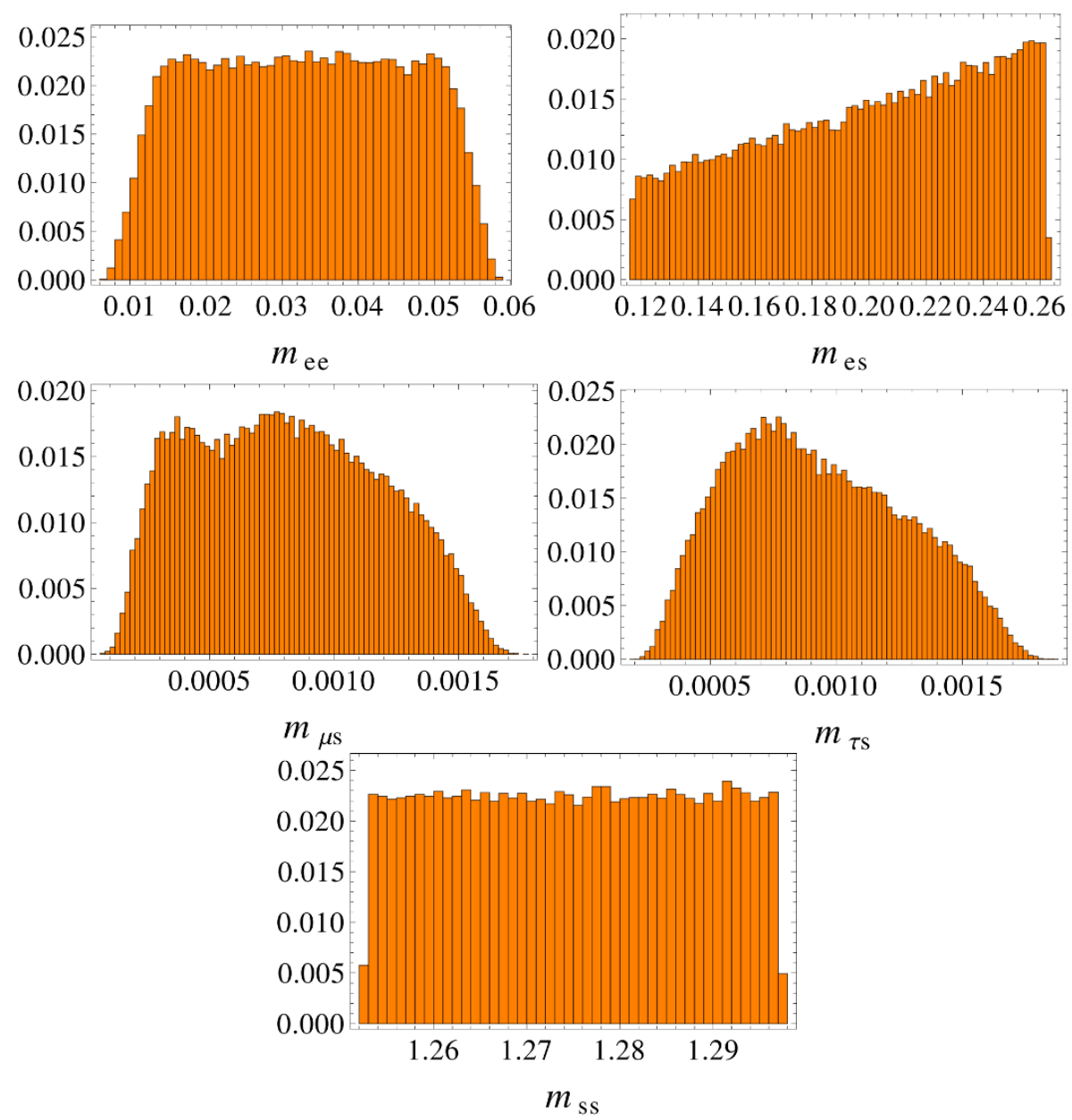

Figure 10. PDFs for the distributions of the absolute value of $\left|m_{e e}\right|,\left|m_{e s}\right|,\left|m_{\mu s}\right|,\left|m_{\tau s}\right|$ and $\left|m_{s s}\right|$, for the normal hierarchy and $\Delta m_{41}^{2}=1.71 \mathrm{eV}^{2}$.

point a view, new data by Planck should be soon released, with a measurement of the number of light species. From the oscillation point of view, a part of the currently allowed parameter space (that allows for a solution to the reactor and Gallium anomalies) will be probed in the next couple of years by the NUCIFER [45] and STEREO [46] experiments. A much broader portion should be explored with a timescale of more than ten years by the updated version of KamLAND, CeLAND [47], in principle allowing to confirm or rule out the presence of a sterile neutrino independently from the cosmological measurements.

\section{Final discussion and conclusion}

In this paper we have analyzed in a probabilistic way the possible structures and correlations among the neutrino mass matrix elements in the standard neutrino oscillation framework, in view of the latest global analysis of the neutrino oscillation data. This is done by constructing PDFs for each matrix element by assuming gaussian distributions for the known oscillation parameters and flat distributions for the unknown ones.

We analyzed the possible textures of the mass matrix allowed by data in the hierarchical and almost degenerate cases and discussed the future perspectives for better determining 

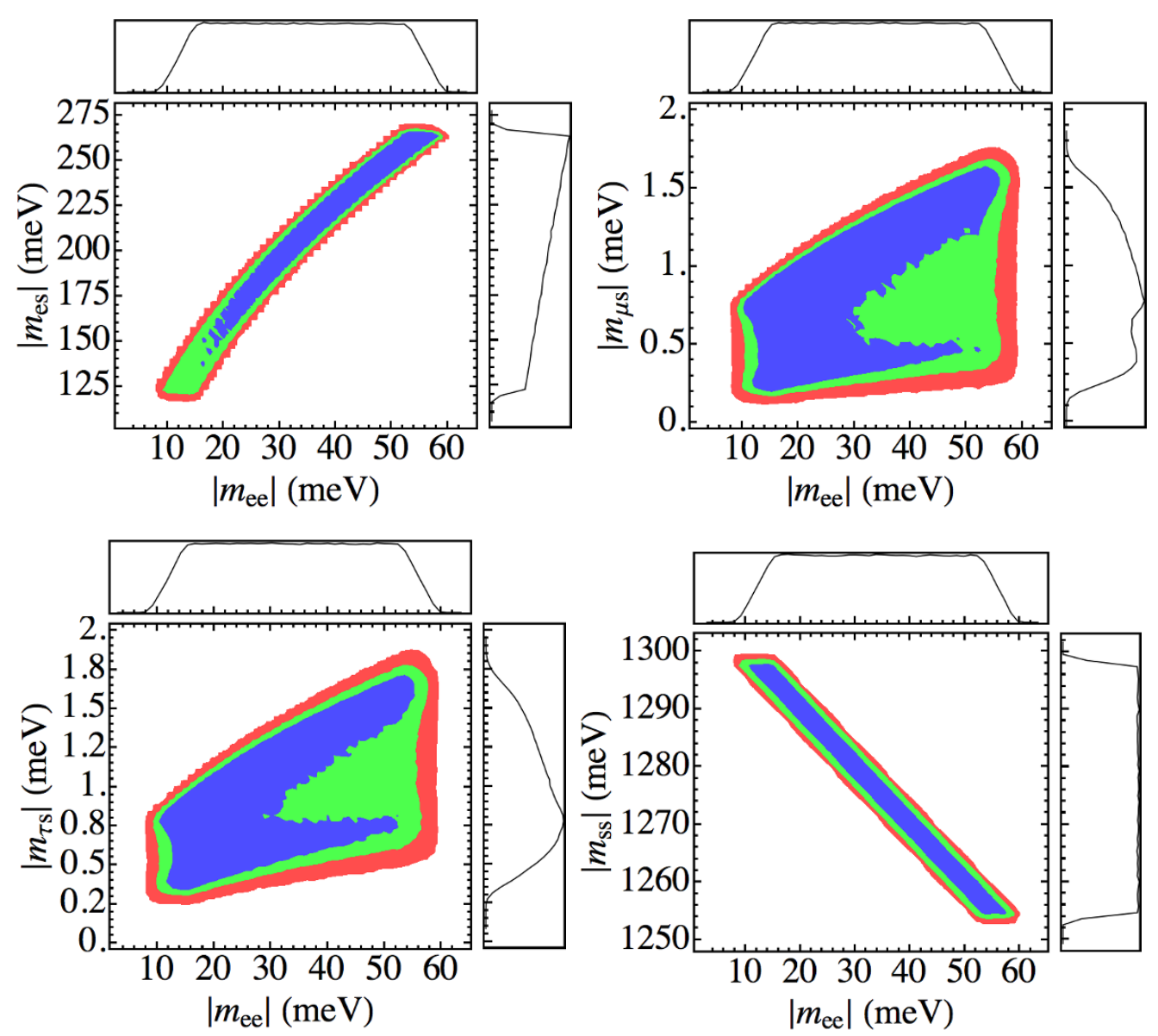

Figure 11. PDFs for the distributions of the absolute values $\left|m_{e e}\right| \times\left|m_{e s}\right|,\left|m_{e e}\right| \times\left|m_{\mu s}\right|,\left|m_{e e}\right| \times$ $\left|m_{\tau s}\right|$ and $\left|m_{e e}\right| \times\left|m_{s s}\right|$, for the normal hierarchy and $\Delta m_{41}^{2}=1.71 \mathrm{eV}^{2}$.

these matrix elements by future neutrino oscillation and non-oscillation data. The conclusion is that a better determination of the currently measured oscillation parameters will, in general, have a small effect on the matrix elements. The biggest effect will come from a better determination of $\sin ^{2} \theta_{23}$ solving the octant degeneracy, as one could have guessed. A determination of $\delta$ would be significant, particularly for the normal hierarchy. In the inverted ordering, it would play a bigger role in the determination of $\left|m_{\mu \tau}\right|$ and $\left|m_{\tau \tau}\right|$. For the quasi-degenerate case, the impact of the determination of $\delta$ is rather small due to the presence of more relevant $\mathrm{CP}$ phases. Future inputs from beta and neutrinoless double beta decay experiments, as well as cosmology, seem to be the most promising in providing new clues to understand the flavor structure. A specially encouraging scenario would be to have $m_{\beta}$ in the reach of KATRIN experiment, the neutrino mass hierarchy settled by near future oscillation experiments, and also a possible cosmological measurement of the sum of neutrino masses.

Models of neutrino mixing based on discrete flavor symmetries, as discussed at the end of in section 3, anticipate relations among mixing angles and the $\delta$ phase that can be tested in the future. If these relations turn out to be true, they will impose correlations among the mass matrix elements beyond the ones considered here. 

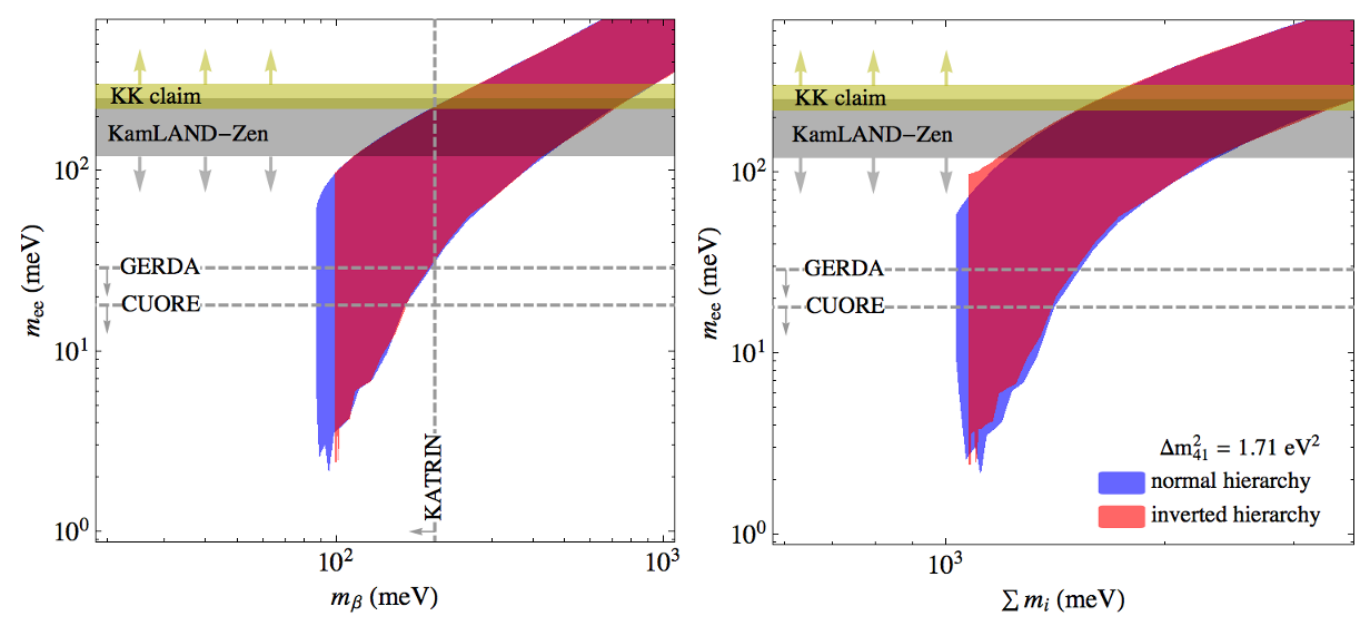

Figure 12. We show the current allowed regions for $\left|m_{e e}\right|$ at $99 \%$ CL as a function of the effective electron neutrino mass, $m_{\beta}$, on the left panels and as a function of the sum of the neutrino masses, $\sum m_{i}$, on the right panels for the $3+1$ scenario. Here $\Delta m_{41}^{2}=1.71 \mathrm{eV}^{2}$. The region allowed by the normal (inverted) mass ordering is in blue (red), and the recent limit on $\left|m_{e e}\right|$ given by KamLANDZen [39] in gray. We also show the reach expected for the beta decay experiment Katrin [42], as well as the ultimate reach aimed by the neutrinoless double beta decay experiments GERDA and CUORE according to ref. [40].

We extend our analysis to include the possibility of a sterile neutrino with mass and mixings allowed by the reactor and gallium anomalies (disregarding current cosmological bounds). We discuss what are the modifications to the mass matrix pattern in this scenario, finding only relevant modifications for $m_{e e}$ as expected. Despite the presence of more parameters than in the standard scenario, the larger sterile neutrino mass would make easier to measure both $\left|m_{e e}\right|$ and $m_{\beta}$. This may also have a big impact in cosmological models, as well as in the future strategies for oscillation experiments.

\section{Acknowledgments}

This work was supported by Fundação de Amparo à Pesquisa do Estado de São Paulo (FAPESP), Conselho Nacional de Desenvolvimento Científico e Tecnológico (CNPq), by the European Commission under the contract PITN-GA-2009-237920 and by the Agence National de la Recherche under contract ANR 2010 BLANC 0413 01. R.Z.F. acknowledges partial support from the European Union FP7 ITN INVISIBLES (Marie Curie Actions, PITN- GA-2011- 289442).

\section{A Matrix elements squared}

Here we give some approximate expressions for the matrix elements $\left|m_{\alpha \beta}\right|^{2}$. We use the following notation: $x^{2}=\sin ^{2} \theta_{12}, y^{2}=\sin ^{2} \theta_{13}, z^{2}=\sin ^{2} \theta_{23}$ and $w^{2}=\sin ^{2} \theta_{14}$. Here we set $\sqrt{1-y^{2}} \rightarrow 1$ and $\sqrt{1-w^{2}} \rightarrow 1$. The standard case is recovered by taking $w \rightarrow 0$.

$$
\begin{aligned}
\left|m_{e e}\right|^{2} \approx & m_{4}^{2} w^{4}+m_{2}^{2} x^{4}+m_{1}^{2}\left(1-x^{2}\right)^{2}+m_{3}^{2} y^{4} \\
& +2 m_{1} m_{4}\left(1-x^{2}\right) w^{2} \cos \left[2\left(\lambda_{1}-\lambda_{4}\right)\right]
\end{aligned}
$$




$$
\begin{aligned}
& +2 m_{1} m_{2} x^{2}\left(1-x^{2}\right) \cos \left(2 \lambda_{1}\right) \\
& +2 m_{1} m_{3} y^{2}\left(1-x^{2}\right) \cos \left[2\left(\delta-\lambda_{1}+\lambda_{3}\right)\right] \\
& +2 m_{2} m_{4} x^{2} w^{2} \cos \left(2 \lambda_{4}\right) \\
& +2 m_{2} m_{3} x^{2} y^{2} \cos \left[2\left(\delta+\lambda_{3}\right)\right] \\
& +2 m_{3} m_{4} y^{2} w^{2} \cos \left[2\left(\delta+\lambda_{3}-\lambda_{4}\right)\right] \\
& \left|m_{e \mu}\right|^{2} \approx\left(1-x^{2}\right) x^{2}\left(1-z^{2}\right)\left(m_{1}^{2}+m_{2}^{2}\right)+\left[m_{1}^{2}\left(1-x^{2}\right)^{2} y^{2}+m_{2}^{2} x^{4} y^{2}+m_{3}^{2} y^{2}\right] z^{2} \\
& +\left[2 m_{1} m_{2} x^{2} y^{2} z^{2}\left(1-x^{2}\right)-2 m_{1} m_{2} x^{2}\left(1-z^{2}\right)\left(1-x^{2}\right)\right] \cos \left(2 \lambda_{1}\right) \\
& -2 m_{1} m_{3} y^{2} z^{2}\left(1-x^{2}\right) \cos \left[2\left(\delta-\lambda_{1}+\lambda_{3}\right)\right] \\
& +\left[2 m_{1}^{2} x y z\left(1-x^{2}\right)^{3 / 2} \sqrt{1-z^{2}}-2 m_{2}^{2} x^{3} y z \sqrt{1-x^{2}} \sqrt{1-z^{2}}\right] \cos \delta \\
& -2 m_{1} m_{2} x y z\left(1-x^{2}\right)^{3 / 2} \sqrt{1-z^{2}} \cos \left(\delta-2 \lambda_{1}\right) \\
& -2 m_{2} m_{3} x^{2} y^{2} z^{2} \cos \left[2\left(\delta+\lambda_{3}\right)\right] \\
& +2 m_{2} m_{3} x y z \sqrt{1-x^{2}} \sqrt{1-z^{2}} \cos \left(\delta+2 \lambda_{3}\right) \\
& -2 m_{1} m_{3} x y z \sqrt{1-x^{2}} \sqrt{1-z^{2}} \cos \left(\delta-2 \lambda_{1}+2 \lambda_{3}\right) \\
& +2 m_{1} m_{2} x^{3} y z \sqrt{1-x^{2}} \sqrt{1-z^{2}} \cos \left(\delta+2 \lambda_{1}\right) \\
& \left|m_{e \tau}\right|^{2} \approx\left(1-x^{2}\right) x^{2} z^{2}\left(m_{1}^{2}+m_{2}^{2}\right)+\left[m_{1}^{2}\left(1-x^{2}\right)^{2} y^{2}+m_{2}^{2} x^{4} y^{2}+m_{3}^{2} y^{2}\right]\left(1-z^{2}\right) \\
& +\left[2 m_{1} m_{2} x^{2} y^{2}\left(1-z^{2}\right)\left(1-x^{2}\right)-2 m_{1} m_{2} x^{2} z^{2}\left(1-x^{2}\right)\right] \cos \left(2 \lambda_{1}\right) \\
& -2 m_{1} m_{3} y^{2}\left(1-z^{2}\right)\left(1-x^{2}\right) \cos \left[2\left(\delta-\lambda_{1}+\lambda_{3}\right)\right] \\
& -\left[2 m_{1}^{2} x y z\left(1-x^{2}\right)^{3 / 2} \sqrt{1-z^{2}}-2 m_{2}^{2} x^{3} y z \sqrt{1-x^{2}} \sqrt{1-z^{2}}\right] \cos \delta \\
& +2 m_{1} m_{2} x y z\left(1-x^{2}\right)^{3 / 2} \sqrt{1-z^{2}} \cos \left(\delta-2 \lambda_{1}\right) \\
& -2 m_{2} m_{3} x^{2} y^{2}\left(1-z^{2}\right) \cos \left[2\left(\delta+\lambda_{3}\right)\right] \\
& -2 m_{2} m_{3} x y z \sqrt{1-x^{2}} \sqrt{1-z^{2}} \cos \left(\delta+2 \lambda_{3}\right) \\
& +2 m_{1} m_{3} x y z \sqrt{1-x^{2}} \sqrt{1-z^{2}} \cos \left(\delta-2 \lambda_{1}+2 \lambda_{3}\right) \\
& -2 m_{1} m_{2} x^{3} y z \sqrt{1-x^{2}} \sqrt{1-z^{2}} \cos \left(\delta+2 \lambda_{1}\right) \\
& \left|m_{\mu \mu}\right|^{2} \approx 4\left(1-z^{2}\right) x^{2} y^{2} z^{2}\left(1-x^{2}\right)\left(m_{1}^{2}+m_{2}^{2}\right)+\left(1-z^{2}\right)^{2}\left[m_{1}^{2} x^{4}+m_{2}^{2}\left(1-x^{2}\right)^{2}\right] \\
& +\left[m_{1}^{2}\left(1-x^{2}\right)^{2} y^{4}+m_{2}^{2} x^{4} y^{4}+m_{3}^{2}\right] z^{4} \\
& +2 m_{1} m_{2} x^{2}\left(1-x^{2}\right)\left[\left(1-z^{2}\right)^{2}-4 y^{2} z^{2}\left(1-z^{2}\right)+y^{4} z^{4}\right] \cos \left(2 \lambda_{1}\right) \\
& +2 m_{1} m_{2}\left(1-z^{2}\right) x^{4} y^{2} z^{2} \cos \left[2\left(\delta+\lambda_{1}\right)\right] \\
& +2 m_{1} m_{2}\left(1-z^{2}\right)(1-x)^{2}(1+x)^{2} y^{2} z^{2} \cos \left[2\left(\delta-\lambda_{1}\right)\right] \\
& +2 m_{2} m_{3}\left(1-z^{2}\right)\left(1-x^{2}\right) z^{2} \cos \left(2 \lambda_{3}\right) \\
& +2\left(m_{1}^{2}+m_{2}^{2}\right)\left(1-z^{2}\right)\left(1-x^{2}\right) x^{2} y^{2} z^{2} \cos (2 \delta) \\
& +4 m_{1} m_{3} \sqrt{1-z^{2}} \sqrt{1-x^{2}} x y z^{3} \cos \left(\delta-2 \lambda_{1}+2 \lambda_{3}\right) \\
& -4 m_{2} m_{3} \sqrt{1-z^{2}} \sqrt{1-x^{2}} x y z^{3} \cos \left(\delta+2 \lambda_{3}\right) \\
& +4 m_{1} m_{2} x^{3} y z \sqrt{1-z^{2}} \sqrt{1-x^{2}}\left[y^{2} z^{2}-\left(1-z^{2}\right)\right] \cos \left(\delta+2 \lambda_{1}\right) \\
& -4 x y z \sqrt{1-x^{2}} \sqrt{1-z^{2}}\left[m_{1}^{2}\left(x^{2}\left(\left(y^{2}+1\right) z^{2}-1\right)-y^{2} z^{2}\right)\right.
\end{aligned}
$$




$$
\begin{aligned}
& \left.+m_{2}^{2}\left(x^{2}\left(\left(y^{2}+1\right) z^{2}-1\right)-z^{2}+1\right)\right] \cos (\delta) \\
& +4 m_{1} m_{2} x y z \sqrt{1-z^{2}} \sqrt{1-x^{2}}\left(1-x^{2}\right)\left[1-\left(1+y^{2}\right) z^{2}\right] \cos \left(\delta-2 \lambda_{1}\right) \\
& +2 m_{2} m_{3} x^{2} y^{2} z^{4} \cos \left[2\left(\delta+\lambda_{3}\right)\right] \\
& +2 m_{1} m_{3}\left(1-x^{2}\right) y^{2} z^{4} \cos \left[2\left(\delta-\lambda_{1}+\lambda_{3}\right)\right] \\
& +2 m_{1} m_{3}\left(1-z^{2}\right) x^{2} z^{2} \cos \left[2\left(\lambda_{1}-\lambda_{3}\right)\right] \\
& \left|m_{\mu \tau}\right|^{2} \approx\left(1-z^{2}\right) z^{2}\left[m_{3}^{2}+\left(1-x^{2}\right)^{2}\left(m_{1}^{2} y^{4}+m_{2}^{2}\right)+x^{4}\left(m_{1}^{2}+m_{2}^{2} y^{4}\right)\right] \\
& +\left(1-x^{2}\right) x^{2} y^{2}\left(m_{1}^{2}+m_{2}^{2}\right)\left[\left(1-z^{2}\right)^{2}+z^{4}\right] \\
& +2 m_{1} m_{2} x^{2}\left[z^{2}\left(1-z^{2}\right)\left(\left(1+y^{2}\right)^{2}-x^{2}\right)-y^{2}\left(1-x^{2}\right)\left(z^{4}+\left(1-z^{2}\right)^{2}\right)\right] \cos \left(2 \lambda_{1}\right) \\
& +2 x y z\left(1-2 z^{2}\right) \sqrt{1-z^{2}} \sqrt{1-x^{2}}\left[\left(1-x^{2}\right)\left(m_{2}^{2}+m_{1}^{2} y^{2}\right)-x^{2}\left(m_{1}^{2}+m_{2}^{2} y^{2}\right)\right] \cos \delta \\
& -2 m_{1} m_{2} x y z\left(1-2 z^{2}\right) \sqrt{1-z^{2}}\left(1-x^{2}\right)^{3 / 2}\left(1+y^{2}\right) \cos \left(\delta-2 \lambda_{1}\right) \\
& +2 m_{1} m_{3} x y z\left(1-2 z^{2}\right) \sqrt{1-z^{2}} \sqrt{1-x^{2}} \cos \left(\delta-2 \lambda_{1}+2 \lambda_{3}\right) \\
& -2 m_{2} m_{3} x y z\left(1-2 z^{2}\right) \sqrt{1-z^{2}} \sqrt{1-x^{2}} \cos \left(\delta+2 \lambda_{3}\right) \\
& -2\left(m_{1}^{2}+m_{2}^{2}\right)\left(1-z^{2}\right)\left(1-x^{2}\right) x^{2} y^{2} z^{2} \cos (2 \delta) \\
& -2 m_{2} m_{3}\left(1-z^{2}\right)\left(1-x^{2}\right) z^{2} \cos \left(2 \lambda_{3}\right) \\
& +2 m_{1} m_{2} x^{3} y z\left(1-2 z^{2}\right) \sqrt{1-z^{2}} \sqrt{1-x^{2}}\left(1+y^{2}\right) \cos \left(\delta+2 \lambda_{1}\right) \\
& -2 m_{1} m_{2} y^{2} z^{2}\left(1-z^{2}\right)\left(1-x^{2}\right)^{2} \cos \left[2\left(\delta-\lambda_{1}\right)\right] \\
& -2 m_{1} m_{2} x^{4} y^{2} z^{2}\left(1-z^{2}\right) \cos \left[2\left(\delta+\lambda_{1}\right)\right] \\
& +2 m_{2} m_{3} x^{2} y^{2} z^{2}\left(1-z^{2}\right) \cos \left[2\left(\delta+\lambda_{3}\right)\right] \\
& +m_{1} m_{3} y^{2}\left(1-x^{2}\right) \cos \left[2\left(\delta-\lambda_{1}+\lambda_{3}\right)\right] \\
& -2 m_{1} m_{3}\left(1-z^{2}\right) x^{2} z^{2} \cos \left[2\left(\lambda_{1}-\lambda_{3}\right)\right] \\
& \left|m_{\tau \tau}\right|^{2} \approx 4\left(1-z^{2}\right) x^{2} y^{2} z^{2}\left(1-x^{2}\right)\left(m_{1}^{2}+m_{2}^{2}\right)+z^{4}\left[m_{1}^{2} x^{4}+m_{2}^{2}\left(1-x^{2}\right)^{2}\right] \\
& +\left[m_{1}^{2}\left(1-x^{2}\right)^{2} y^{4}+m_{2}^{2} x^{4} y^{4}+m_{3}^{2}\right]\left(1-z^{2}\right)^{2} \\
& +2 m_{1} m_{2} x^{2}\left(1-x^{2}\right)\left[y^{4}\left(1-z^{2}\right)^{2}-4 y^{2} z^{2}\left(1-z^{2}\right)+z^{4}\right] \cos \left(2 \lambda_{1}\right) \\
& +2 m_{1} m_{2}\left(1-z^{2}\right) x^{4} y^{2} z^{2} \cos \left[2\left(\delta+\lambda_{1}\right)\right] \\
& +2 m_{1} m_{2}\left(1-z^{2}\right)(1-x)^{2}(1+x)^{2} y^{2} z^{2} \cos \left[2\left(\delta-\lambda_{1}\right)\right] \\
& +2 m_{2} m_{3}\left(1-z^{2}\right)\left(1-x^{2}\right) z^{2} \cos \left(2 \lambda_{3}\right) \\
& +2\left(m_{1}^{2}+m_{2}^{2}\right)\left(1-z^{2}\right)\left(1-x^{2}\right) x^{2} y^{2} z^{2} \cos (2 \delta) \\
& -4 m_{1} m_{3} \sqrt{1-z^{2}} \sqrt{1-x^{2}} x y z\left(1-z^{2}\right) \cos \left(\delta-2 \lambda_{1}+2 \lambda_{3}\right) \\
& +4 m_{2} m_{3} \sqrt{1-z^{2}} \sqrt{1-x^{2}} x y z\left(1-z^{2}\right) \cos \left(\delta+2 \lambda_{3}\right) \\
& +4 m_{1} m_{2} x^{3} y z \sqrt{1-z^{2}} \sqrt{1-x^{2}}\left[y^{2} z^{2}-y^{2}+z^{2}\right] \cos \left(\delta+2 \lambda_{1}\right) \\
& -4 x y z \sqrt{1-x^{2}} \sqrt{1-z^{2}}\left[m_{1}^{2}\left(\left(1-x^{2}\right) y^{2}\left(1-z^{2}\right)+x^{2} z^{2}\right)\right. \\
& \left.-m_{2}^{2}\left(x^{2} y^{2}\left(1-z^{2}\right)+z^{2}\left(1-x^{2}\right)\right)\right] \cos (\delta) \\
& +4 m_{1} m_{2} x y z \sqrt{1-z^{2}} \sqrt{1-x^{2}}\left(1-x^{2}\right)\left[\left(1-z^{2}\right) y^{2}-z^{2}\right] \cos \left(\delta-2 \lambda_{1}\right) \\
& +2 m_{2} m_{3} x^{2} y^{2}(1-z)^{2}(1+z)^{2} \cos \left[2\left(\delta+\lambda_{3}\right)\right] \\
& +2 m_{1} m_{3}\left(1-x^{2}\right) y^{2}(1-z)^{2}(1+z)^{2} \cos \left[2\left(\delta-\lambda_{1}+\lambda_{3}\right)\right]
\end{aligned}
$$




$$
\begin{aligned}
& +2 m_{1} m_{3}\left(1-z^{2}\right) x^{2} z^{2} \cos \left[2\left(\lambda_{1}-\lambda_{3}\right)\right] \\
& \left|m_{s e}\right|^{2} \approx w^{2}\left[m_{2}^{2} x^{4}+m_{1}^{2}\left(1-x^{2}\right)^{2}+m_{3}^{2} y^{4}+m_{4}^{2}\right] \\
& -2 m_{3} m_{4} w^{2} y^{2} \cos \left[2\left(\delta+\lambda_{3}-\lambda_{4}\right)\right] \\
& +2 m_{1} m_{2} w^{2} x^{2}\left(1-x^{2}\right) \cos \left(2 \lambda_{1}\right) \\
& +2 m_{1} m_{3} w^{2} y^{2}\left(1-x^{2}\right) \cos \left[2\left(\delta-\lambda_{1}+\lambda_{3}\right)\right] \\
& -2 m_{1} m_{4} w^{2}\left(1-x^{2}\right) \cos \left[2\left(\lambda_{1}-\lambda_{4}\right)\right] \\
& +2 m_{2} m_{3} w^{2} x^{2} y^{2} \cos \left[2\left(\delta+\lambda_{3}\right)\right] \\
& -2 m_{2} m_{4} w^{2} x^{2} \cos \left(2 \lambda_{4}\right) \\
& \left|m_{s \mu}\right|^{2} \approx w^{2}\left[m_{1}^{2}\left(x^{2}-1\right)\left(x^{2}\left(\left(y^{2}+1\right) z^{2}-1\right)-y^{2} z^{2}\right)\right. \\
& \left.+m_{2}^{2} x^{2}\left(x^{2}\left(\left(y^{2}+1\right) z^{2}-1\right)-z^{2}+1\right)+m_{3}^{2} y^{2} z^{2}\right] \\
& +2 w^{2} x y z \sqrt{1-x^{2}} \sqrt{1-z^{2}}\left[\left(1-x^{2}\right) m_{1}^{2}-x^{2} m_{2}^{2}\right] \cos \delta \\
& -2 m_{1} m_{2} w^{2} x y z\left(1-x^{2}\right)^{3 / 2} \sqrt{1-z^{2}} \cos \left(\delta-2 \lambda_{1}\right) \\
& +2 m_{1} m_{2} w^{2} x^{3} y z \sqrt{1-x^{2}} \sqrt{1-z^{2}} \cos \left(\delta+2 \lambda_{1}\right) \\
& -2 m_{1} m_{3} w^{2} x y z \sqrt{1-x^{2}} \sqrt{1-z^{2}} \cos \left(\delta-2 \lambda_{1}+2 \lambda_{3}\right) \\
& +2 m_{2} m_{3} w^{2} x y z \sqrt{1-x^{2}} \sqrt{1-z^{2}} \cos \left(\delta+2 \lambda_{3}\right) \\
& -2 m_{2} m_{3} w^{2} x^{2} y^{2} z^{2} \cos \left[2\left(\delta+\lambda_{3}\right)\right] \\
& +2 m_{1} m_{2} w^{2} x^{2}\left(1-x^{2}\right)\left[\left(1+y^{2}\right) z^{2}-1\right] \cos \left(2 \lambda_{1}\right) \\
& -2 m_{1} m_{3}\left(1-x^{2}\right) w^{2} y^{2} z^{2} \cos \left[2\left(\delta-\lambda_{1}+\lambda_{3}\right)\right] \\
& \left|m_{s \tau}\right|^{2} \approx w^{2}\left[m_{1}^{2}\left(1-x^{2}\right)\left(\left(1-x^{2}\right) y^{2}\left(1-z^{2}\right)+x^{2} z^{2}\right)\right. \\
& \left.+m_{2}^{2}\left(x^{2} z^{2}+x^{4}\left(y^{2}\left(1-z^{2}\right)-z^{2}\right)\right)+m_{3}^{2} y^{2}\left(1-z^{2}\right)\right] \\
& -2 w^{2} x y z \sqrt{1-x^{2}} \sqrt{1-z^{2}}\left[\left(1-x^{2}\right) m_{1}^{2}-x^{2} m_{2}^{2}\right] \cos \delta \\
& +2 m_{1} m_{2} w^{2} x y z\left(1-x^{2}\right)^{3 / 2} \sqrt{1-z^{2}} \cos \left(\delta-2 \lambda_{1}\right) \\
& -2 m_{1} m_{2} w^{2} x^{3} y z \sqrt{1-x^{2}} \sqrt{1-z^{2}} \cos \left(\delta+2 \lambda_{1}\right) \\
& +2 m_{1} m_{3} w^{2} x y z \sqrt{1-x^{2}} \sqrt{1-z^{2}} \cos \left(\delta-2 \lambda_{1}+2 \lambda_{3}\right) \\
& -2 m_{2} m_{3} w^{2} x y z \sqrt{1-x^{2}} \sqrt{1-z^{2}} \cos \left(\delta+2 \lambda_{3}\right) \\
& -2 m_{2} m_{3} w^{2} x^{2} y^{2}\left(1-z^{2}\right) \cos \left[2\left(\delta+\lambda_{3}\right)\right] \\
& -2 m_{1} m_{2} w^{2} x^{2}\left(1-x^{2}\right)\left[z^{2}-\left(1-z^{2}\right) y^{2}\right] \cos \left(2 \lambda_{1}\right) \\
& -2 m_{1} m_{3}\left(1-x^{2}\right) w^{2} y^{2}\left(1-z^{2}\right) \cos \left[2\left(\delta-\lambda_{1}+\lambda_{3}\right)\right] \\
& \left|m_{s s}\right|^{2} \approx w^{4}\left[\left(1-x^{2}\right)^{2} m_{1}^{2}+x^{4} m_{2}^{2}+y^{4} m_{3}^{2}\right]+m_{4}^{2} \\
& +2 m_{1} m_{2} w^{4} x^{2}\left(1-x^{2}\right) \cos \left(2 \lambda_{1}\right) \\
& +2 m_{2} m_{4} w^{4} x^{2} \cos \left(2 \lambda_{4}\right) \\
& +2 m_{1} m_{3} w^{4} y^{2}\left(1-x^{2}\right) \cos \left[2\left(\delta-\lambda_{1}+\lambda_{3}\right)\right] \\
& +2 m_{1} m_{4} w^{2}\left(1-x^{2}\right) \cos \left[2\left(\lambda_{1}-\lambda_{4}\right)\right] \\
& +2 m_{2} m_{3} w^{4} x^{2} y^{2} \cos \left[2\left(\delta+\lambda_{3}\right)\right] \\
& +2 m_{3} m_{4} w^{2} y^{2} \cos \left[2\left(\delta+\lambda_{3}-\lambda_{4}\right)\right]
\end{aligned}
$$


B Complete set of correlation plots for the matrix elements
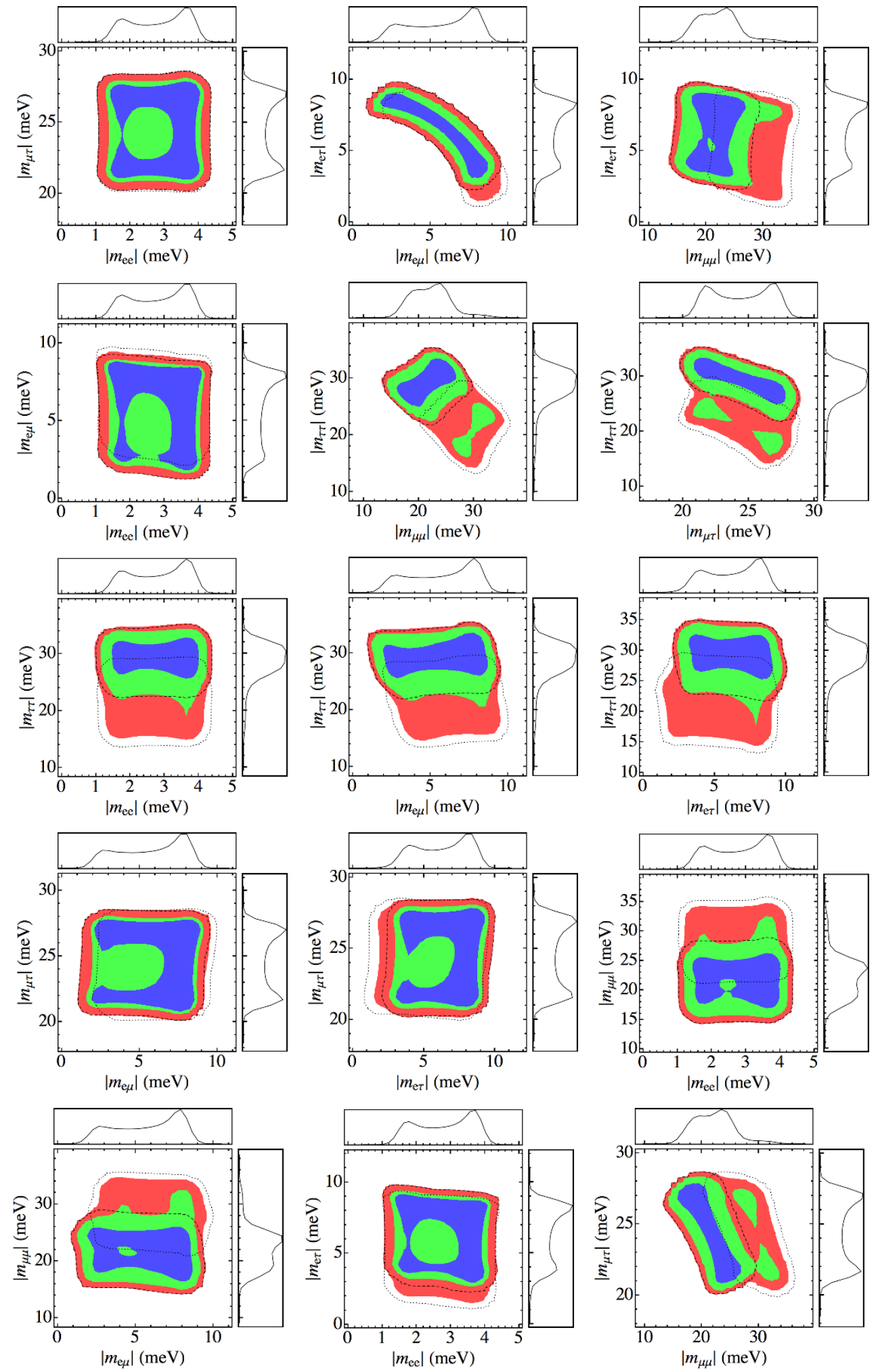

Figure 13. PDFs for the distribution of the absolute value of several pairs of matrix elements. We use blue, green and red for the allowed region at $68.27 \%, 95.45 \%$ and $99.73 \% \mathrm{CL}$, respectively. Here $m_{1} \rightarrow 0$. The dashed (dotted) lines refer to the $99.73 \%$ CL for $\theta_{23}$ in the first (second) octant. 

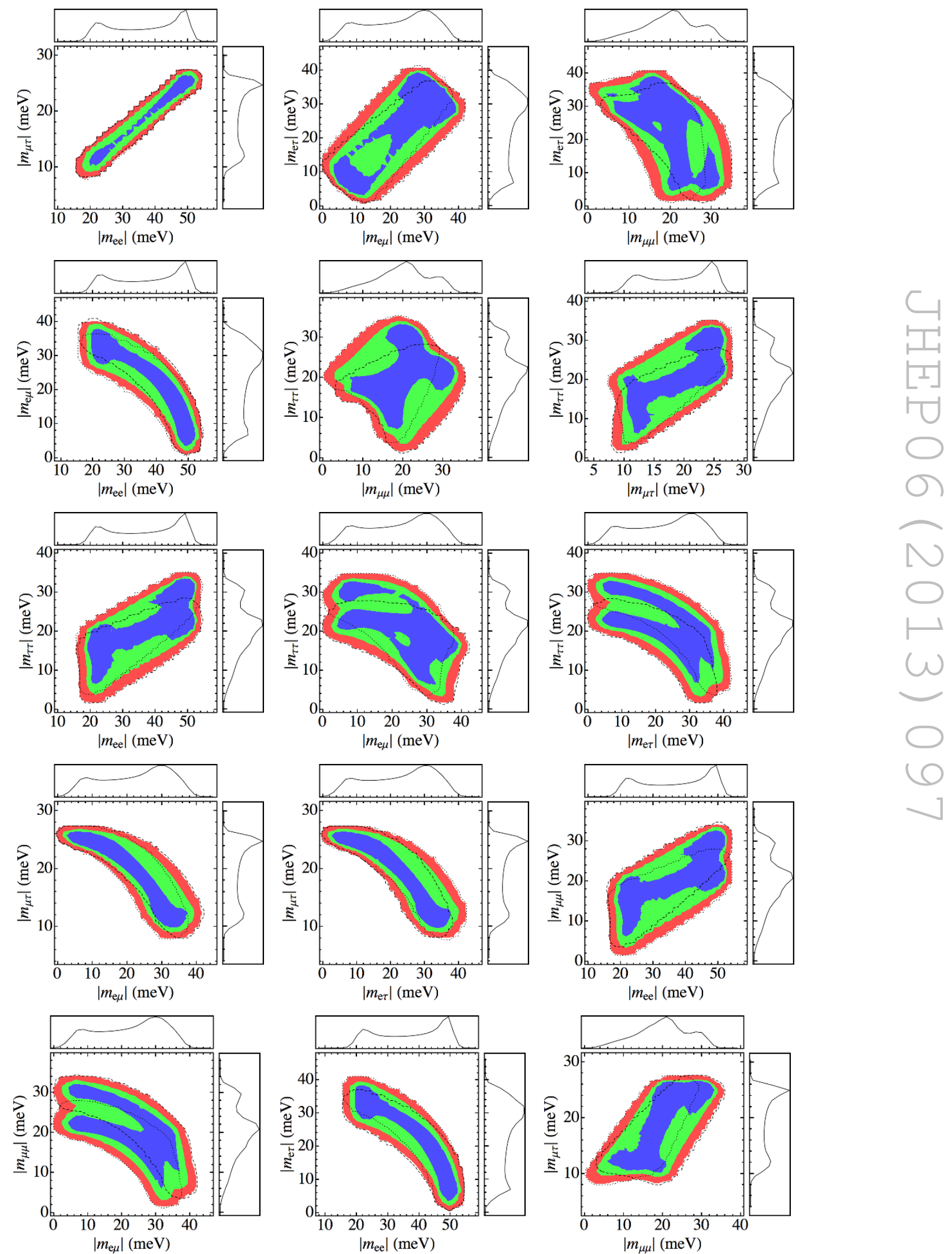

Figure 14. PDFs for the distribution of the absolute value of several pairs of matrix elements. We use blue, green and red for the allowed region at $68.27 \%, 95.45 \%$ and $99.73 \%$ CL, respectively. Here $m_{3} \rightarrow 0$. The dashed (dotted) lines refer to the $99.73 \%$ CL for $\theta_{23}$ in the first (second) octant. 

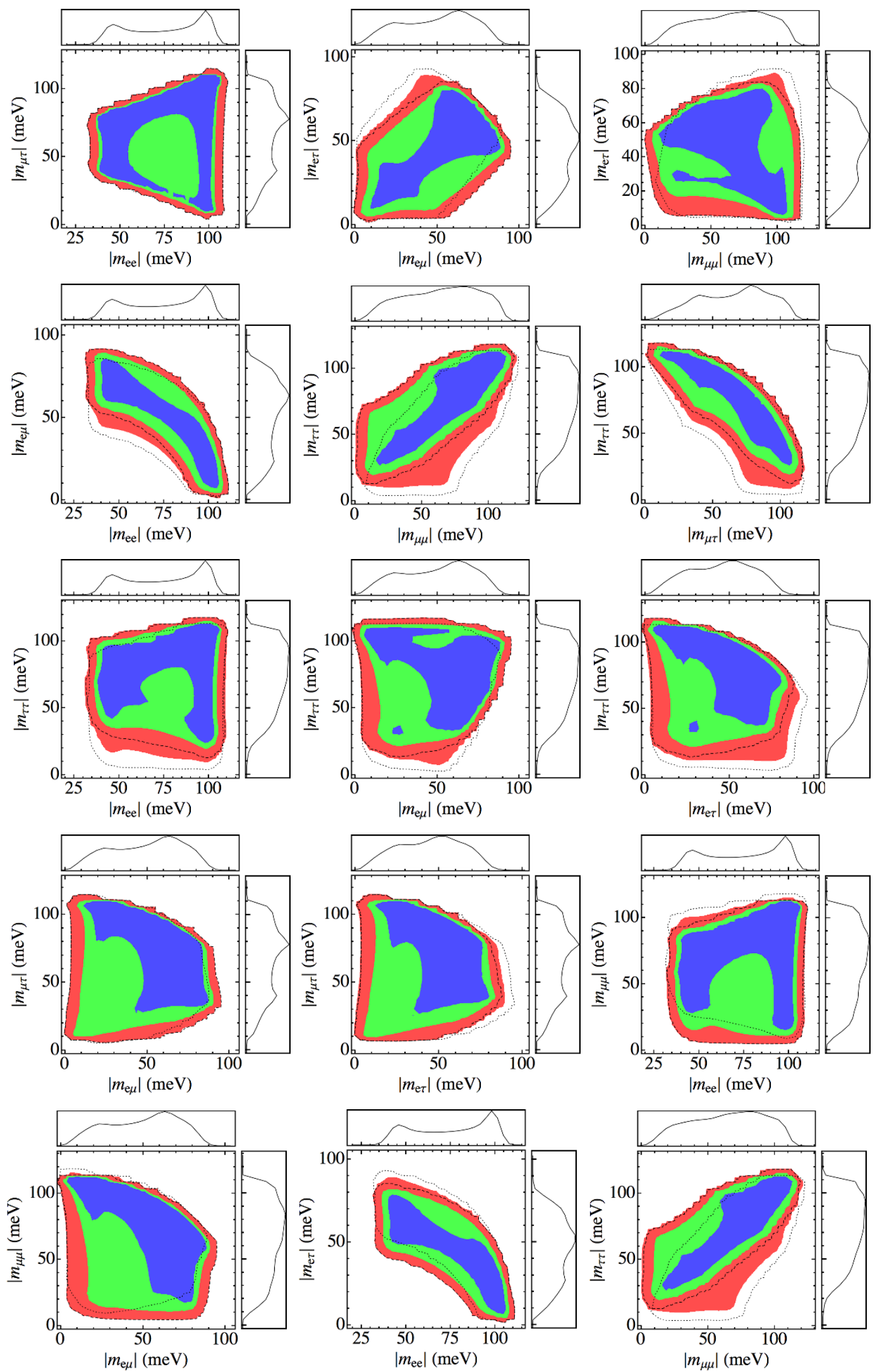

Figure 15. PDFs for the distribution of the absolute value of several pairs of matrix elements. We use blue, green and red for the allowed region at $68.27 \%, 95.45 \%$ and $99.73 \% \mathrm{CL}$, respectively. Here $m_{1} \rightarrow 0$ and we impose the normal mass ordering. The dashed (dotted) lines refer to the $99.73 \%$ CL for $\theta_{23}$ in the first (second) octant. 
Open Access. This article is distributed under the terms of the Creative Commons Attribution License which permits any use, distribution and reproduction in any medium, provided the original author(s) and source are credited.

\section{References}

[1] DOUBLE-CHOOZ collaboration, Y. Abe et al., Indication for the disappearance of reactor electron antineutrinos in the Double CHOOZ experiment, Phys. Rev. Lett. 108 (2012) 131801 [arXiv:1112.6353] [INSPIRE].

[2] DAYA-BAY collaboration, F. An et al., Observation of electron-antineutrino disappearance at Daya Bay, Phys. Rev. Lett. 108 (2012) 171803 [arXiv:1203.1669] [InSPIRE].

[3] RENO collaboration, J. Ahn et al., Observation of Reactor Electron Antineutrino Disappearance in the RENO Experiment, Phys. Rev. Lett. 108 (2012) 191802 [arXiv: 1204.0626] [INSPIRE].

[4] T2K collaboration, K. Abe et al., Indication of Electron Neutrino Appearance from an Accelerator-produced Off-axis Muon Neutrino Beam, Phys. Rev. Lett. 107 (2011) 041801 [arXiv:1106.2822] [INSPIRE].

[5] MINOS collaboration, P. Adamson et al., Improved search for muon-neutrino to electron-neutrino oscillations in MINOS, Phys. Rev. Lett. 107 (2011) 181802 [arXiv:1108.0015] [INSPIRE].

[6] S. Emery, T2K, in Rencontres IPhT/SPP, Paris, France, June 2013.

[7] Y. Takaesu, Determination of the mass hierarchy with medium-baseline reactor-neutrino experiments, arXiv:1304.5306 [INSPIRE].

[8] P. Machado, H. Minakata, H. Nunokawa and R. Zukanovich Funchal, Combining Accelerator and Reactor Measurements of $\theta_{13}$ : The First Result, JHEP 05 (2012) 023 [arXiv:1111.3330] [INSPIRE].

[9] G. Fogli, E. Lisi, A. Marrone, D. Montanino, A. Palazzo et al., Global analysis of neutrino masses, mixings and phases: entering the era of leptonic CP-violation searches, Phys. Rev. D 86 (2012) 013012 [arXiv: 1205.5254] [INSPIRE].

[10] M. Gonzalez-Garcia, M. Maltoni, J. Salvado and T. Schwetz, Global fit to three neutrino mixing: critical look at present precision, JHEP 12 (2012) 123 [arXiv:1209.3023] [INSPIRE].

[11] D. Forero, M. Tortola and J. Valle, Global status of neutrino oscillation parameters after Neutrino-2012, Phys. Rev. D 86 (2012) 073012 [arXiv:1205.4018] [INSPIRE].

[12] LSND collaboration, C. Athanassopoulos et al., Evidence for $\bar{\nu}_{\mu} \rightarrow \bar{\nu}_{e}$ oscillations from the LSND experiment at LAMPF, Phys. Rev. Lett. 77 (1996) 3082 [nucl-ex/9605003] [INSPIRE].

[13] LSND collaboration, A. Aguilar-Arevalo et al., Evidence for neutrino oscillations from the observation of anti-neutrino(electron) appearance in a anti-neutrino(muon) beam, Phys. Rev. D 64 (2001) 112007 [hep-ex/0104049] [INSPIRE].

[14] MiniBoonE collaboration, A. Aguilar-Arevalo et al., Event Excess in the MiniBooNE Search for $\bar{\nu}_{\mu} \rightarrow \bar{\nu}_{e}$ Oscillations, Phys. Rev. Lett. 105 (2010) 181801 [arXiv:1007.1150] [INSPIRE].

[15] GALLEX collaboration, P. Anselmann et al., First results from the Cr-51 neutrino source experiment with the GALLEX detector, Phys. Lett. B 342 (1995) 440 [INSPIRE]. 
[16] GALLEX collaboration, W. Hampel et al., Final results of the Cr-51 neutrino source experiments in GALLEX, Phys. Lett. B 420 (1998) 114 [INSPIRE].

[17] F. Kaether, W. Hampel, G. Heusser, J. Kiko and T. Kirsten, Reanalysis of the GALLEX solar neutrino flux and source experiments, Phys. Lett. B 685 (2010) 47 [arXiv:1001.2731] [INSPIRE].

[18] SAGE collaboration, J. Abdurashitov et al., Measurement of the response of the Russian-American gallium experiment to neutrinos from a Cr-51 source, Phys. Rev. C 59 (1999) 2246 [hep-ph/9803418] [INSPIRE].

[19] J. Abdurashitov, V. Gavrin, S. Girin, V. Gorbachev, P. Gurkina et al., Measurement of the response of a Ga solar neutrino experiment to neutrinos from an Ar-37 source, Phys. Rev. C 73 (2006) 045805 [nucl-ex/0512041] [INSPIRE].

[20] SAGE collaboration, J. Abdurashitov et al., Measurement of the solar neutrino capture rate with gallium metal. III: Results for the 2002-2007 data-taking period, Phys. Rev. C 80 (2009) 015807 [arXiv:0901.2200] [INSPIRE].

[21] T. Mueller, D. Lhuillier, M. Fallot, A. Letourneau, S. Cormon et al., Improved Predictions of Reactor Antineutrino Spectra, Phys. Rev. C 83 (2011) 054615 [arXiv:1101.2663] [InSPIRE].

[22] P. Huber, On the determination of anti-neutrino spectra from nuclear reactors, Phys. Rev. C 84 (2011) 024617 [Erratum ibid. C 85 (2012) 029901] [arXiv: 1106. 0687] [INSPIRE].

[23] G. Mention, M. Fechner, T. Lasserre, T. Mueller, D. Lhuillier et al., The Reactor Antineutrino Anomaly, Phys. Rev. D 83 (2011) 073006 [arXiv:1101.2755] [inSPIRE].

[24] J. Kopp, M. Maltoni and T. Schwetz, Are there sterile neutrinos at the eV scale?, Phys. Rev. Lett. 107 (2011) 091801 [arXiv:1103.4570] [INSPIRE].

[25] C. Giunti and M. Laveder, 3+1 and 3+2 Sterile Neutrino Fits, Phys. Rev. D 84 (2011) 073008 [arXiv: 1107.1452 ] [INSPIRE].

[26] P. Machado, H. Nunokawa, F.P. dos Santos and R.Z. Funchal, Bulk Neutrinos as an Alternative Cause of the Gallium and Reactor Anti-neutrino Anomalies, Phys. Rev. D 85 (2012) 073012 [arXiv:1107.2400] [INSPIRE].

[27] M. Frigerio and A.Y. Smirnov, Structure of neutrino mass matrix and CP-violation, Nucl. Phys. B 640 (2002) 233 [hep-ph/0202247] [INSPIRE].

[28] M. Frigerio and A.Y. Smirnov, Neutrino mass matrix: Inverted hierarchy and CP-violation, Phys. Rev. D 67 (2003) 013007 [hep-ph/0207366] [INSPIRE].

[29] A. Merle and W. Rodejohann, The Elements of the neutrino mass matrix: Allowed ranges and implications of texture zeros, Phys. Rev. D 73 (2006) 073012 [hep-ph/0603111] [INSPIRE].

[30] W. Grimus and P. Ludl, Correlations of the elements of the neutrino mass matrix, JHEP 12 (2012) 117 [arXiv:1209.2601] [INSPIRE].

[31] CHOOZ collaboration, M. Apollonio et al., Search for neutrino oscillations on a long baseline at the CHOOZ nuclear power station, Eur. Phys. J. C 27 (2003) 331 [hep-ex/0301017] [INSPIRE].

[32] MINOS collaboration, P. Adamson et al., Measurement of the neutrino mass splitting and flavor mixing by MINOS, Phys. Rev. Lett. 106 (2011) 181801 [arXiv:1103.0340] [INSPIRE]. 
[33] Y. Lin, Tri-bimaximal Neutrino Mixing from $A_{4}$ and $\theta_{13} \sim \theta_{C}$, Nucl. Phys. B 824 (2010) 95 [arXiv:0905.3534] [INSPIRE].

[34] G. Altarelli, F. Feruglio, L. Merlo and E. Stamou, Discrete Flavour Groups, theta $a_{13}$ and Lepton Flavour Violation, JHEP 08 (2012) 021 [arXiv:1205.4670] [INSPIRE].

[35] M.-C. Chen, J. Huang, J.-M. O'Bryan, A.M. Wijangco and F. Yu, Compatibility of $\theta_{13}$ and the Type I Seesaw Model with $A_{4}$ Symmetry, JHEP 02 (2013) 021 [arXiv:1210.6982] [INSPIRE].

[36] D. Hernandez and A.Y. Smirnov, Lepton mixing and discrete symmetries, Phys. Rev. D 86 (2012) 053014 [arXiv: 1204.0445] [INSPIRE].

[37] D. Hernandez and A.Y. Smirnov, Discrete symmetries and model-independent patterns of lepton mixing, arXiv:1212.2149 [INSPIRE].

[38] P. Coloma, P. Huber, J. Kopp and W. Winter, Systematic uncertainties in long-baseline neutrino oscillations for large $\theta_{13}$, arXiv: 1209.5973 [INSPIRE].

[39] KamLAND-Zen collaboration, A. Gando et al., Limit on Neutrinoless $\beta \beta$ Decay of Xe-136 from the First Phase of KamLAND-Zen and Comparison with the Positive Claim in Ge-76, Phys. Rev. Lett. 110 (2013) 062502 [arXiv:1211.3863] [INSPIRE].

[40] X. Sarazin, Review of double beta experiments, arXiv:1210.7666 [INSPIRE].

[41] K. Abazajian, E. Calabrese, A. Cooray, F. De Bernardis, S. Dodelson et al., Cosmological and Astrophysical Neutrino Mass Measurements, Astropart. Phys. 35 (2011) 177 [arXiv: 1103.5083] [INSPIRE].

[42] KATRIN collaboration, S. Fischer, Status of the KATRIN experiment, PoS (EPS-HEP2011) 097.

[43] A. Belesev, A. Berlev, E. Geraskin, A. Golubev, N. Likhovid et al., An upper limit on additional neutrino mass eigenstate in 2 to $100 \mathrm{eV}$ region from 'Troitsk nu-mass' data, JETP Lett. 97 (2013) 67 [arXiv:1211.7193] [INSPIRE].

[44] M. Cirelli, G. Marandella, A. Strumia and F. Vissani, Probing oscillations into sterile neutrinos with cosmology, astrophysics and experiments, Nucl. Phys. B 708 (2005) 215 [hep-ph/0403158] [INSPIRE].

[45] http://indico.cern.ch/getFile.py/access?contribId=6\&sessionId=7\&resId= 0\&materialId $=$ slides\&confId $=207406$.

[46] http://indico.cern.ch/getFile.py/access?contribId=7\&sessionId=7\&resId= $0 \&$ materialId $=$ slides\&confI $=207406$.

[47] M. Cribier, M. Fechner, T. Lasserre, A. Letourneau, D. Lhuillier et al., A proposed search for a fourth neutrino with a PBq antineutrino source, Phys. Rev. Lett. 107 (2011) 201801 [arXiv:1107.2335] [INSPIRE]. 